\title{
Assessing Dynamic Models for High Priority Waste Collection in Smart Cities
}

\author{
Theodoros Anagnostopoulos 1,2 , Kostas Kolomvatsos 3 , Christos Anagnostopoulos 4 , \\ Arkady Zaslavsky 2,5 , and Stathes Hadjiefthymiades ${ }^{3}$ \\ 1 Community Imaging Group, University of Oulu, 90570, Finland; tanagnos@ee.oulu.fi \\ 2 Department of Infocommunication Technologies, ITMO University, 197101, Russia \\ 3 Department of Informatics and Telecommunications, University of Athens, 15784, Greece; \\ \{kostasks, shadj\}@di.uoa.gr \\ 4 School of Computing Science, University of Glasgow, G12 8QQ, UK; christos.anagnostopoulos@glasgow.ac.uk \\ 5 CSIR0 Computational Informatics, CSIR0, Box 312, Vic. 3169, Australia; arkady.zaslavsky@csiro.au
}

\begin{abstract}
A B S T R A C T
Waste Management (WM) represents an important part of Smart Cities (SCs) with significant impact on modern societies. WM involves a set of processes ranging from waste collection to the recycling of the collected materials. The proliferation of sensors and actuators enable the new era of Internet of Things (IoT) that can be adopted in SCs and help in WM. Novel approaches that involve dynamic routing models combined with the IoT capabilities could provide solutions that outperform existing models. In this paper, we focus on a SC where a number of collection bins are located in different areas with sensors attached to them. We study a dynamic waste collection architecture, which is based on data retrieved by sensors. We pay special attention to the possibility of immediate WM service in high priority areas, e.g., schools or hospitals where, possibly, the presence of dangerous waste or the negative effects on human quality of living impose the need for immediate collection. This is very crucial when we focus on sensitive groups of citizens like pupils, elderly or people living close to areas where dangerous waste is rejected. We propose novel algorithms aiming at providing efficient and scalable solutions to the dynamic waste collection problem through the management of the trade-off between the immediate collection and its cost. We describe how the proposed system effectively responds to the demand as realized by sensor observations and alerts originated in high priority areas. Our aim is to minimize the time required for serving high priority areas while keeping the average expected performance at high level. Comprehensive simulations on top of the data retrieved by a SC validate the proposed algorithms on both quantitative and qualitative criteria which are adopted to analyze their strengths and weaknesses. We claim that, local authorities could choose the model that best matches their needs and resources of each city.
\end{abstract}

Keywords: Internet of Things; Smart Cities; Dynamic Models; High Priority Areas; Waste Collection

\section{Introduction}

In modern societies, the increased population accompanied by the industrial development leads to a boost of economies. Booming economies, rapid urbanization and the rise in community living standards have greatly accelerated the waste generation rate in developing countries (Minghua et al., 2009). Through this perspective, Waste Management (WM) is a critical issue for every modern society/city. The reason is that waste should be efficiently managed in order to minimize its negative effects in the environment and, thus, to increase the quality of life for citizens. Local authorities or private companies can undertake the responsibility to provide a high quality mechanism for WM. In the past, important improvements have been observed in WM. Related research has identified the relevant stakeholders and organizations that may have an interest in adequate WM. For instance, some of the reported stakeholders are: national or local governments, municipal authorities, city corporations, non-governmental organizations, households, private contractors, Ministries of Health, Environment, Economy and Finance, recycling and waste processing companies.

The WM process involves a number of issues ranging from the collection of waste to the recycling. Waste Management Systems (WMSs) are devoted to provide functionalities that effectively handle the lifecycle of various types of waste. Information and Communication Technologies (ICT) can offer many advantages when incorporated in WMSs. Sensors and actuators enable the new era of Internet of Things (IoT) that can be adopted in Smart Cities (SCs) and help in WM. The provision of intelligent applications that control the entire line of WM based on sensor observations facilitates the necessary processes and maximizes the performance. A SC could deploy a number of sensors attached to waste bins in order to gather/collect data related to waste (e.g., weight, odour). A central system could have a view on the 
waste information realized 'in-the-field' and, thus, it could be able to take the appropriate decisions related to the demand for waste generation/collection. In addition, such system could be responsive when alerts are triggered in real time. For instance, the system could re-arrange the routes of collection trucks, when necessary, leading to a dynamic WM scheme.

Prior research focuses on the collection, transfer and transport practices and has proposed the appropriate strategies for collection schemes, route planning, collection schedule and the appropriate infrastructure or the number of the required resources for waste collection. However, some important issues are still open. For instance, there is the need of adopting effective methodologies for the management of: (a) dynamic changes in the production of waste and (b) how cities affect WM. Actually, these two issues are related to how and when waste is produced and what are the appropriate solutions for its efficient management in real time. Societies need an intelligent framework that dynamically responds to changes in the production of waste especially when waste is produced in critical (high priority) areas. As a critical (high priority) area in a city, we could define areas that are mostly affected by waste, especially, when the collection process is not frequently performed. There are specific types of waste that should be immediately collected and recycled due to the negative effects that they have in humans' lives. A representative example involves an area where specific amenities are located like schools, hospitals, university campuses, etc. In such areas, waste bins close to the discussed amenities should be immediately depleted. In addition, high priority areas could be also characterized areas where 'sensitive' groups of people are living (e.g., people living close to hospitals or fuel stations). Waste bins located in such areas could be characterized as high priority bins. High priority bins are related to: (i) waste dangerous for human lives (e.g., chemicals) or (ii) sensitive areas that are heavily affected by waste disposals (e.g., schools, gas stations); such areas are characterized either by the type of the amenities located in them or by the type of people living at them. In both cases, such bins should be depleted as soon as possible in order to minimize the effect of waste into the environment and the human lives. For instance, hospitals' waste should be immediately collected to minimize the risk of exposing humans to chemicals or other medical-related materials. Bins close to gas stations should also be immediately collected to minimize the risk of fire. The immediate collection of waste in high priority areas becomes imperative when no special process is applied for recycling dangerous materials.

In this paper, we present a WM framework to be adopted by a SC.

Definition (Bakici et al., 2013). A SC is a high-tech intensive and advanced city that connects people, information and city elements using new technologies in order to create a sustainable, greener city, competitive and innovative commerce, and an increased life quality.

Aiming to the increased quality of life, the proposed framework is responsible for deriving dynamic decisions for the efficient collection of waste especially for the management of high priority bins. The proposed system provides routing functionalities for a number of trucks and offers routes adaptation when waste collection needs are identified in high priority areas. The priority of each area is defined according to the type of the area, however, the system could be easily extended and rely on top of different constraints. An intelligent mechanism undertakes the responsibility of dynamically adapting the route for each collection truck when waste bins, in high priority areas, are full. Hence, the system gives priority to sensitive areas, thus, maximizing the quality of life for citizens together with the maximization of the performance for waste collection. We propose a set of collection strategies realized into four (4) models for facing the aforementioned scenario. Each strategy has specific characteristics concerning the method that the system adopts to manage high priority bins. The aim is to provide a set of solutions for the efficient collection of the high priority waste bins in a SC. We perform a large number of simulations in order to reveal the advantages of each model and present comprehensive evaluation results. Our aim is to provide a comparison between the proposed models and, accordingly, stakeholders can easily select one of them according to the special needs of each area. The following list reports on the contributions of our work:

- we adopt of the notion of high priority areas and high priority bins, respectively, in WM;

- we propose four (4) WM models for serving the immediate collection of high priority bins;

- we provide routing functionalities and routing adaptation for serving areas that are characterized as critical (high priority);

- the proposed models manage the trade-off between the immediate collection and the cost for waste depletion; 
- the proposed framework dynamically responds to changes in the production of waste in high priority areas;

- we provide a comprehensive experimental evaluation of the proposed models that reveal their strengths and weaknesses over a large set of simulation scenarios.

We focus on areas that are mainly affected by waste disposal. Some examples of areas and amenities that could be characterized as high priority are: (i) hospitals (e.g., people can be exposed to medical-related materials), (ii) schools (e.g., pupils or students can be considered as 'sensitive' target group as far as the waste disposal consequences concerns), (iii) areas close to fuel stations (e.g., there is an increased risk of fire, especially in areas where high temperatures are observed), (iv) areas close to factories that utilize materials not supported by special plans for immediate treatment (e.g., in the case where no specific individual collection and recycling processes are present); (v) areas that, for specific reasons, the local authorities want to be managed as high priority (e.g., squares, places where people are gathered, playgrounds); In these cases, the seasonal aspects could be also applied (a location could be of high priority only for a specific time interval, e.g., various events, touristic areas).

The paper is organized as follows. Section 2 presents related research efforts while in Section 3, we discuss the proposed framework. We analytically describe the system and give its main characteristics. In Section 4, we describe the proposed models for managing high priority waste bins and in Section 5, we describe the application perspectives of our framework. In Section 6, we report on the performance of each model. We compare the proposed models for important performance metrics for waste management. Finally, in Section 7, we conclude our paper by giving future research directions.

\section{Prior Work}

A number of dynamic models for waste collection have been proposed by the research community. There is a significant interest for dynamic models since static approaches cannot handle the dynamic nature of IoT potentiality. The dynamic scheduling and routing model discussed in (Johansson, 2006) adopts capacity sensors and wireless communication infrastructure, thus, it is able to be aware of each bin's state. The mechanism incorporates analytical modeling and discrete-event simulation in order to achieve real-time dynamic routing and scheduling. The authors in (Wy et al., 2013) introduce a rollon-rolloff routing, serving multiple disposal facilities, with huge amounts of waste at construction sites and shopping districts. The model adopts large neighborhood search with iterative heuristic algorithms. In (Nuortio et al., 2006), an improved dynamic route planning is discussed. The authors enhance a guided variable neighborhood threshold meta-heuristic adapted to the problem of waste collection. The authors in (Reed et al., 2014) propose a model which incorporates the Ant Colony System (ACS) in order to achieve dynamic routing. The authors treat the location of bins as a spatial network and apply the $k$-means algorithm in order to cluster the bins into a set of partial clusters.

In (Zsigraiova et al., 2013), the authors combine routing and scheduling optimization. Historical data applied to individual bins establish the daily circuits of collection points to be visited. Planning is applied to scheduling for better system management. The authors in (Li et al., 2008) consider dynamic scheduling over a set of previously defined collection paths. The main objective of the approach is to minimize the total operational and fixed costs for collection trucks. In (Nadizadeh \& Nasab, 2014), the authors introduce a dynamic routing model based on fuzzy demands by assuming the demands of the customers as fuzzy variables. The presented model incorporates a heuristic approach based on fuzzy credibility theory. A mathematical formulation methodology is proposed in (Ramos et al., 2014) for the development of a plan of service areas, defining routing and scheduling. The model takes into consideration possible new alternative solutions as it manages the system as a whole. In (Buhrkal et al., 2012), the authors propose routing with time windows which analyze the logistics activity within a city. The proposed mechanism finds the cost of optimal routes in order to guide the trucks to bins with an adaptive large neighborhood search algorithm. In (Stellingwerff, 2011), the authors evaluate dynamic planning methods applied for waste collection of underground bins. The proposed model reduces the amounts of carbon dioxide released in the environment by making the dynamic routing more effective. Discrete event simulation is the technique adopted in (Mes, 2012). The authors present a model that applies dynamic planning to exploit information transmitted through motion sensors embedded in underground bins. The authors in (Milić \& Jovanović, 2011) develop a routing algorithm with a mobile measuring system on the trucks. They perform stochastic dynamic routing which makes corrections during or after the execution of the existing routes. 
The authors in (Minh et al., 2013) introduce a memetic algorithm to perform routing enforced with time windows and conflicts context. The model incorporates a combination of flow and set partitioning formulation to achieve multi-objective optimization. Another heuristic solution is proposed in (Hemmelmayr et al., 2013). The authors state the waste collection as a periodic truck routing problem with intermediate waste depots. The model incorporates variable neighborhood search and dynamic programming in order to achieve the optimal solution. In (Von Poser \& Awad, 2006), the authors propose a genetic algorithm to solve the dynamic routing problem. Specifically, the model assumes that the waste collection problem could be treated as a Traveling Salesman Problem (TSP). Then, the genetic algorithm optimally solves the TSP. The authors in (Mes et al., 2013) propose a heuristic method for the dynamic routing considering several tunable parameters. Sensors enable reverse inventory routing in dense waste networks. As a waste network, they consider a network of waste bins located inside a specific city. Heuristics deal with uncertainty of daily and seasonal effects. The model discussed in (Bing, 2014) deals with the collection of plastic waste which is differentiated from other solid waste. Collection routes are redesigned by adopting an eco-efficiency metric with balancing the trade-off between the costs and environmental issues. In (Anagnostopoulos and Zaslavsky, 2014), the authors propose a novel IoT-enabled dynamic routing model for waste collection in a SC. The proposed model is robust in case of an emergency (i.e., a road under construction, unexpected traffic congestion). Finally, the authors in (Anagnostopoulos et al., 2015a) extend the system presented in (Anagnostopoulos \& Zaslavsky, 2014) and propose a dynamic routing model to face the case of truck inefficiency due to overloading or damage. The paper incorporates IoT technology applied for waste collection in a SC. In (Anagnostopoulos \& Zaslavsky, 2014; Anagnostopoulos et al., 2015a), the waste collection is addressed as a problem which can be solved with IoT infrastructure incorporated in SCs.

\section{Significance of Our Research}

As described, relevant research in WM mainly involves solutions related to collection strategies. These strategies involve routing and/or scheduling algorithms that deliver the optimal paths/schedules for waste collection. The ultimate goal is the minimization of the operational cost. The related work efforts deal with the problem through the economic perspective of the WM problem. Hence, many efforts provide solutions for the dynamic scheduling and routing when deviations from the initial plan are present. In this paper, we go a step further. We study the problem not only through the economic perspective; however, we consider the problem as a major problem that affects human lives. We consider high priority areas and high priority waste bins, respectively. Our aim is to provide high quality solutions for the management of high priority bins when there is a need for the immediate collection. Our approach differs with previous models in the following aspect. In (Anagnostopoulos \& Zaslavky, 2014), the authors focus on the design of a dynamic routing algorithm capable of handling collection truck routing due to road abnormalities (e.g., under construction) or unexpected traffic congestion. In (Anagnostopoulos et al., 2015a), the authors extend the algorithm proposed in (Anagnostopoulos \& Zaslavsky, 2014) by incorporating the management of the truck inefficiency due to overloading or damages during the collection process. In this paper, the proposed models are based on a dynamic routing algorithm which is an extension of the previous work in (Anagnostopoulos \& Zaslavsky, 2014). The main difference of the current paper is our view to extend the dynamic routing process in immediately collecting high priority bins paying attention of keeping the humans' quality of life at high levels. Conceptually, this means that the main dynamic routing concerns have been addressed by previous approaches and, now, we focus on the models applied in a more advanced concept on WM, i.e., by increasing the quality of life for citizens within the SC.

\section{System Overview}

Waste collection is a major counterpart to the environmental pollution (Nam \& Pardo, 2012). In the respective literature, waste collection is treated uniformly regarding city areas. However, real situations imply the discrimination of city areas according to certain social criteria, such as: (i) sensitivity to pollution due to medical waste (i.e., in hospitals), (ii) quality of service to a specific population (i.e., in tourist areas), (iii) prestigious places and buildings within the city (i.e., in the municipality town hall), etc. These areas require time critical waste collection. In this paper, we introduce a novel approach of discriminating city areas by incorporating high priority bins to them.

We consider that a SC is divided to a number of sectors $s_{i}, i=1,2, \ldots, n$. Sectors cover the entire area of the city. In each $s_{i}$, a number of 'regular' bins $b_{j}, j=1,2$, ..., $m$ and a certain number of high priority bins $h_{z}, z=$ $1,2, \ldots, p$ are located (Centre of Regional Science, 2015; Priano \& Guerra, 2014). In addition, each sector is served by a number of trucks $t_{l}, l=1,2, \ldots, q$. It holds 
that the number of high priority bins in a sector is less than the number of regular bins since high priority areas are, usually, less than regular areas within a sector.

Each bin, regardless its type, has certain features, such as:

- a static GPS location;

- RFIDs for bin tagging and identification;

- capacity sensors for measuring the volume of waste;

- actuators for locking the lid of the bin when becomes full;

- $\quad$ a volume capacity of $c$ kilograms to serve waste.

Each collection truck has certain features as follows:

- a dynamic GPS location which changes on the move;

- a volume capacity of $C$ kilograms to collect waste from the bins.

It holds that the capacity of each truck is much more than the capacity of a bin, thus, $C \gg c$.

Waste collection is the process of collecting waste from the bins and empties it to the dump outside the SC. We propose four (4) waste collection models, which deliver a realization of a waste collection strategy. Such strategy serves regular or high priority bins, in a daily basis, susceptible to the restriction that collection is discriminated according to the bin type. Consequently, high priority bins are served immediately when they get full (e.g., their capacity status is over a pre-defined threshold to avoid frequent, possibly costly depletions) interpolating the truck routing trip between regular bins. The proposed models are dynamic in the sense that they react when changes in the demand for waste collection are present. For instance, when bins become full, specific events are triggered in the backend system and, accordingly, changes in the trucks routes are imposed in order to immediately serve the raised alerts.

The immediate collection, especially for high priority bins, is imperative, even though, bins have lids to be locked when they are full. The reason is that by locking the bins the problem is not solved since future waste disposals (that could be realized in short time after locking) will, probably, contaminate the area near the locked bins. Let us discuss a specific scenario where the significance of the proposed models becomes clear. Imagine a hospital where the corresponding bins are locked. In this case, future (possibly dangerous) waste disposals (e.g., medical - related material) could heavily affect human lives. Obviously, when bins are collected once a day, this could possibly cause many problems to patients, employees or visitors. The proposed models minimize the time required for collecting high priority bins in order to minimize possible negative effects in humans.

\section{System Architecture}

The architecture of the system is applied to the concept of IoT-enabled SCs (Vermesan \& Friess, 2013; Jin et al., 2014). It is composed of three layers:

(i) The physical infrastructure,

(ii) The middleware available in the Cloud, and

(iii) The Decision Support System (DSS).

The physical infrastructure contains the devices embedded to bins and trucks. Data produced by the IoT components of bins are fused to a Wireless Sensor Network (WSN) in order to be transferred to the central system for further processing. Trucks, apart from the GPS location tracking device, are equipped by smart phones used by drivers for getting routing directions. The Cloud middleware is built on top of the OpenIoT ${ }^{1}$. Specifically, WSN data are aggregated from the Global Sensor Networks (GSN) ${ }^{2}$ which is originated in the lower level of the OpenIoT. Consequently, data are cleaned and missing values are imputed. Data are stored in a Cloud Data Base (Cloud DB). The DB also stores GPS location data retrieved by trucks. A dynamic scheduling model is responsible to initiate a route when waste in a bin reaches a certain capacity threshold. Accordingly, a dynamic routing model is triggered to produce the appropriate route for collecting waste, as described in Section 4. The DSS is built on top of the architecture and is responsible for: (i) sending routing directions to drivers through an Android GUI, and (ii) producing reports and statistics for the municipality stakeholders; thus, enabling online monitoring of the waste collection process. The proposed architecture is presented in Fig. 1.

The main difference, between the static models proposed in the literature and the dynamic models proposed in this paper, is the treatment of waste collection according to the filling rates of the bins. In (Anagnostopoulos et al., 2015b) a dynamic scheduling model for is presented for the efficient waste collection based on top- $k$ queries. Specifically, the authors experiment on dynamic vs. static scheduling; with regards to waste collection time, for different number of $k$ bins. The dynamic model is running continuously

\footnotetext{
${ }^{1}$ https://github.com/OpenIotOrg/openiot

${ }^{2}$ http://lsir.epfl.ch/research/current/gsn/
} 
while the static model is executed every 24 hours. It can be observed that scheduling time varies w.r.t. the value of top- $k$ bins, thus, the higher the $k$ value, the more time (i.e., logarithmically) required to initiate a schedule. Note that the highest scheduling time is reached for the static scheduling; since in this case, there is no information about the capacity of the collected bins (i.e., full or half full), thus, leading to a low system performance. As reported, through simulations, in (Anagnostopoulos et al., 2015b), a significant number of bins become full in 1-12 hours. It should be noted that in (Anagnostopoulos et al., 2015b) no high priority bins are considered and the lower the $k$ is, the greater the filling rate becomes. In this paper, the proposed models incorporate a dynamic scheduling approach and continually run during the collection process. It should be noted that, at first, the initial routing plans are created when the system is started and accordingly the proposed models are fired to 'monitor' and respond to changes in the demand for collection.
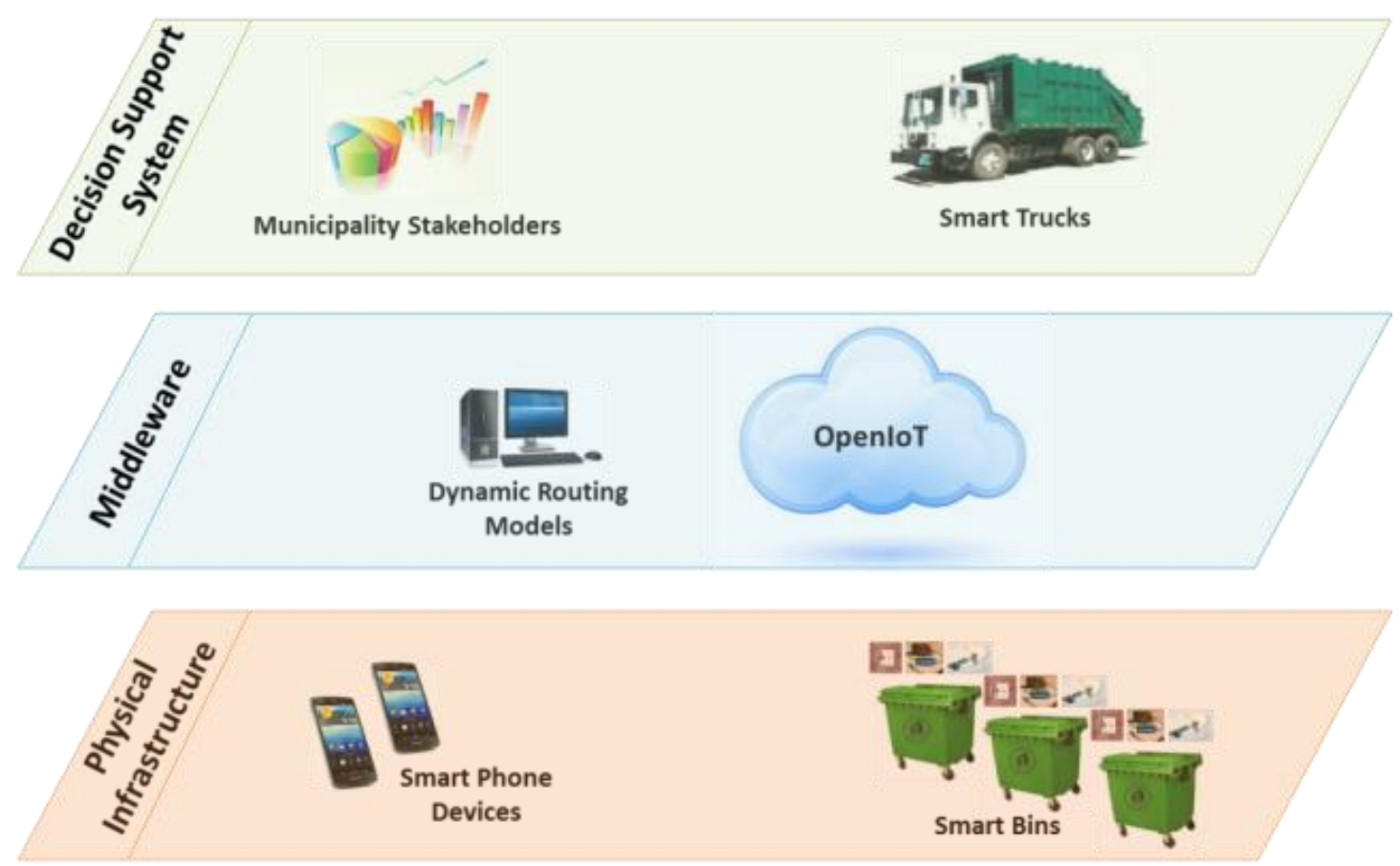

Fig. 1. The system architecture.

\section{High Priority Waste Collection Models}

High priority waste collection involves the immediate response to alerts related to waste bins located in high priority areas. Such alerts are triggered when a high priority bin becomes full or its filling rate is over to a pre-defined threshold. In this paper, we propose a set of models for the immediate management of high priority bins. The proposed models are applied after the generation of the routes that collection trucks should follow to perform the waste collection for regular bins. This means that our models are responsible to provide reactions in high priority bins alerts during the collection process. Hence, in the beginning, the system produces the routes for each collection truck and, accordingly, it initiates (one of) the proposed algorithms (models) to respond with the optimal reaction during the collection process.

It is worth noting that the proposed models could be easily extended to be applied in generic waste collection schemes. For instance, routing plans could be re-adjusted during the collection process in order to better respond to new needs for waste collection. When an area is not participating in the initial collection plan and the need for collection is generated during the execution of the initial plan, the system, based on the proposed models, could readjust the routing plan in order to serve the new areas. However, in such cases, the system should be capable of meeting specific constraints related to trucks load, etc. The initial plans are created by incorporating constraints related to e.g., the trucks 
load. Deviations could make the trucks become full before the end of the trip and, thus, more changes in the plans of the fleet are required.

The simplest (baseline) model includes specific collection trucks devoted to exclusively serve high priority bins. Hence, when an alert is present, one of the devoted trucks undertakes the responsibility of serving the specific bin. The remaining models deal with dynamic routing and scheduling solutions during the collection time. With the proposed models, we aim to handle cases where high priority bins become full during the day and, more specifically, during the collection time. We aim to handle cases where we are not sure in advance when and how high priority waste bins will become full e.g., cases met in commercial blocks, schools, hospitals, crowded touristic areas, etc. In other words, we try to cover unexpected scenarios as far as waste production concerns. In this section, we analytically present the proposed models and give their details. We provide a set of solutions and present their advantages and disadvantages. Hence, developers or local authorities, according to the characteristics of each model, can adopt the model that best matches to a set of pre-defined constraints. In short, the proposed models are:

- the Dedicated Trucks Model (DTM)

- the Detour Model (DM)

- the Minimum Distance Model (MDM)

- the Reassignment Model (RM)

\section{A. The Dedicated Trucks Model (DTM)}

The DTM is the simplest model for high priority waste bins management. In this model, we devote specific trucks for serving high priority bins. The trucks are not assigned to any other collection action. When alarms for full high priority bins are triggered, one of the available trucks undertakes the responsibility of serving them according to its capabilities. In the case of a large number of high priority bins alerts, multiple trucks could be used. It should be noted that the optimal number of trucks should be adopted in order to cover the highest possible number of high priority bins. The DTM will be adopted when local authorities desire immediate responses to high priority bins alerts without disturbing the remaining collection trucks from their initial assignments. The DTM could be the ideal solution when local authorities face a high rate of alerts initiated by high priority bins. However, when no such alerts are present, the performance of the model is low. For instance, if just one high priority bin becomes full during a day, the algorithm will devote a specific truck for this bin. Even worse, multiple routes could be generated when high priority bins (having similar locations) produce alerts with low rate during the day.

The DTM algorithm is depicted in Fig. 2 and results the route $r$ (i.e., the ordered set of waste bins to get emptied) of a specific dedicated truck. The input of the algorithm is the set of high priority bins $h_{z}$ in a specific area and the available trucks $t_{l}$ (i.e., the trucks devoted to serve high priority bins - here $t_{l}$ represents the number of trucks devoted to high priority bins and not the number of trucks devoted to a specific sector). The output is the route $r$ for a truck devoted to the collection of the discussed bins. A route $r$ is a sequence of waste bins that a truck should visit. The algorithm, through the adoption of the routing function (i.e., routing()), utilizes the Dijsktra shortest path to generate the initial route for visiting the high priority bins. The visited () function is adopted to generate the bins that are visited according to the route $r$ (this is depicted by the set $v$ ). Finally, when a new bin becomes full (its capacity $c_{h_{z}}$ is over a threshold $\theta$ ) during the collection process, the algorithm excludes the visited bins and performs a re-routing process starting over, however, for the remaining bins (depicted by the set difference $r-v)$.

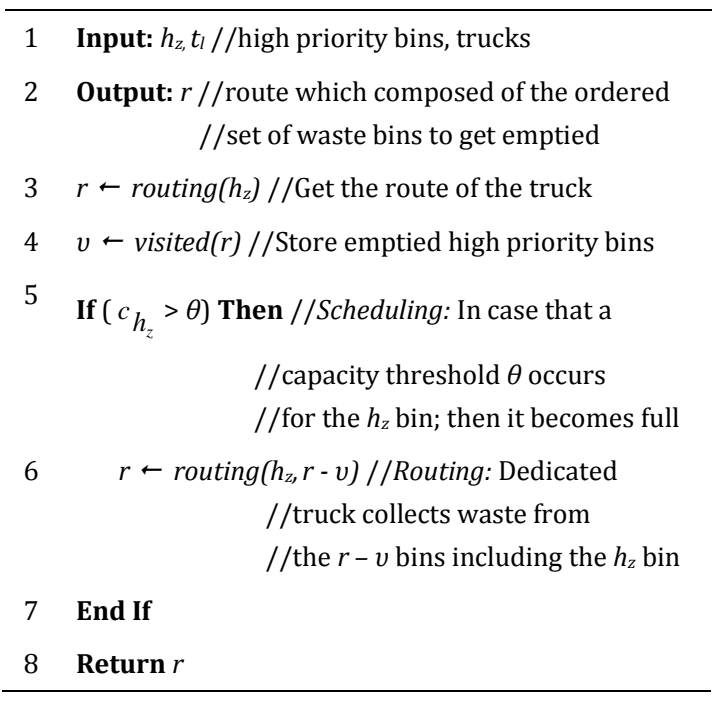

Fig. 2. The DTM algorithm.

The time complexity of the DTM is the complexity of the routing 0 function, which is $\mathrm{O}\left(h_{z}^{2}\right)$, plus the complexity of the visited ( function which is $\mathrm{O}\left(h_{z}\right)$. Hence, the overall complexity is $\mathrm{O}\left(h_{z}^{2}\right)$. 


\section{B. The Detour Model (DM)}

As the DTM does not exhibit good performance when high priority bins alert are relatively rare, we propose the DM. This model forces trucks to deviate from their original path in order to first serve the high priority bins that have already triggered an alert. The routing function of DM incorporates an initialization function which indicates that the routing will start from the location of a specified high priority bin. Each collection truck is responsible to change its root to serve the wider area where high priority bins are located. For instance, let us consider that the original allocation for two trucks $T_{1}, T_{2}$, is two sub-areas, A1, A2 for each truck respectively Fig. 3. If an alarm is triggered by a high priority bin located in $\mathrm{A} 1$, the first truck, $\mathrm{T}_{1}$, will interrupt its route, it will serve the bin and, accordingly it will continue with the remaining bins. The DM aims to have the trucks devoted to specific sub-areas. Every bin inside each area will be served by the corresponding truck. However, the model exhibits low performance when the truck, which is responsible for high priority bin, is located in a distant place related to the location of the bin. In such cases, there is the risk of producing continuous spatial deviations from the original path which could lead to increased route distances and fuel consumption.

The proposed DM algorithm is depicted in Fig. 4 and results the route of a specific truck. The input of the algorithm is the set of high priority bins $h_{z}$ in a specific sub-area, the set of 'regular' bins $b_{j}$ and the available trucks $t_{l}$. The output is related to the route $r$ for a truck serving the specific area. The algorithm, through the adoption of the routing function (i.e., routing()), utilizes the Dijsktra shortest path to generate the initial route for visiting the 'regular' bins. The visited() function is adopted to generate the bins that are visited according to the path $r$ (this is depicted by the set $v$ ). When a high priority bin becomes full (its capacity $c_{h_{z}}$ is over a threshold $\theta$ ) during the collection process, the algorithm excludes the visited bins and performs a re-routing process. However, the route, with the help of the init() function, starts from the full high priority bin generating the alert and the remaining bins (depicted by the set $r-v$ ) follow.

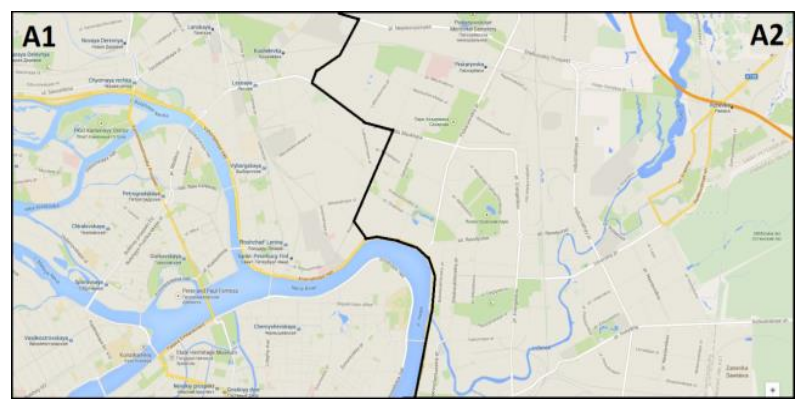

Fig. 3. An allocation example for two trucks.

1 Input: $b_{j}, h_{z} t_{l} / /$ regular bins, high priority bins, $/ /$ trucks

2 Output: $r / /$ route which composed of the ordered //set of waste bins to get emptied

$3 r \leftarrow \operatorname{routing}\left(b_{j}\right) / /$ Get the route of the truck

$4 \quad v \leftarrow \operatorname{visited}(r) / /$ Store emptied regular bins

5 If $\left(c_{h_{z}}>\theta\right)$ Then //Scheduling: In case that a //certain capacity threshold $\theta$ occurs //for the $h$ bin; then it becomes full

6 $r \leftarrow \operatorname{routing}\left(\operatorname{init}\left(h_{z}\right), r-v\right) / /$ Routing: $/ /$ Truck collects waste from the $r-v$ $/ /$ bins including $h_{z}$ bin. Initial routing //bin is the $h_{z}$ bin

$7 \quad$ End If

8 Return $r$

Fig. 4. The DM algorithm.

The time complexity of the DM is the complexity of the routing $\left(\right.$ function, which is $\mathrm{O}\left(b_{j}^{2}\right)$, plus the complexity of the visited() function, which is $\mathrm{O}\left(b_{j}\right)$, and the complexity of the init() function which is $\mathrm{O}\left(h_{z}\right)$. Hence, the overall complexity is $\mathrm{O}\left(b_{j}^{2}\right)$ since $b_{j}$ $>h_{z}$.

\section{The Minimum Distance Model (MDM)}

The above described models, DTM and DM, have specific disadvantages related to the reduced truck load and the possibility of multiple deviations from the initial route, respectively. We propose an additional model, the MDM, which tries to reduce the risk of deviations violating the initial assignments as produced by the system. In the MDM, when an alert is triggered by a high priority bin, the truck having the minimum distance with the bin is assigned to serve it. Specifically, the MDM incorporates a nearest function, which indicates that routing process and selects the truck with the nearest location to the location of the specified high priority bin. An initialization function is also incorporated in the routing function of the MDM. With this model, we try 
to minimize the effort for each truck accompanied by an on-time service for high priority bins. Actually, we try to immediately serve high priority bins mainly located in the borders of the initial allocated subareas. The model minimizes the risk of deviation from the original routes, however, it is affected by the distribution of the produced alerts.

The MDM algorithm is presented in Fig. 5. The inputs of the algorithm are the sets of regular, high priority bins and the available trucks. The output is the route for each truck. Initially, the algorithm generates the route of each truck based on the function routing $(0$ and defines the visited bins with the help of the function visited $\left(\right.$ set $v_{l}$ ). When a high priority bin becomes full (its capacity $c_{h_{z}}$ is over a threshold $\theta$ ) during the collection process, the algorithm, through the adoption of the function nearest(), finds the nearest truck $f$ to the specific bin and performs a re-routing process for the specific truck. In the re-routing process, the algorithm excludes the already visited bins (let $r_{f}-v_{f}$ be the set of the visited bins) and gives priority (starts the route) from the high priority bin through the use of the function init ().

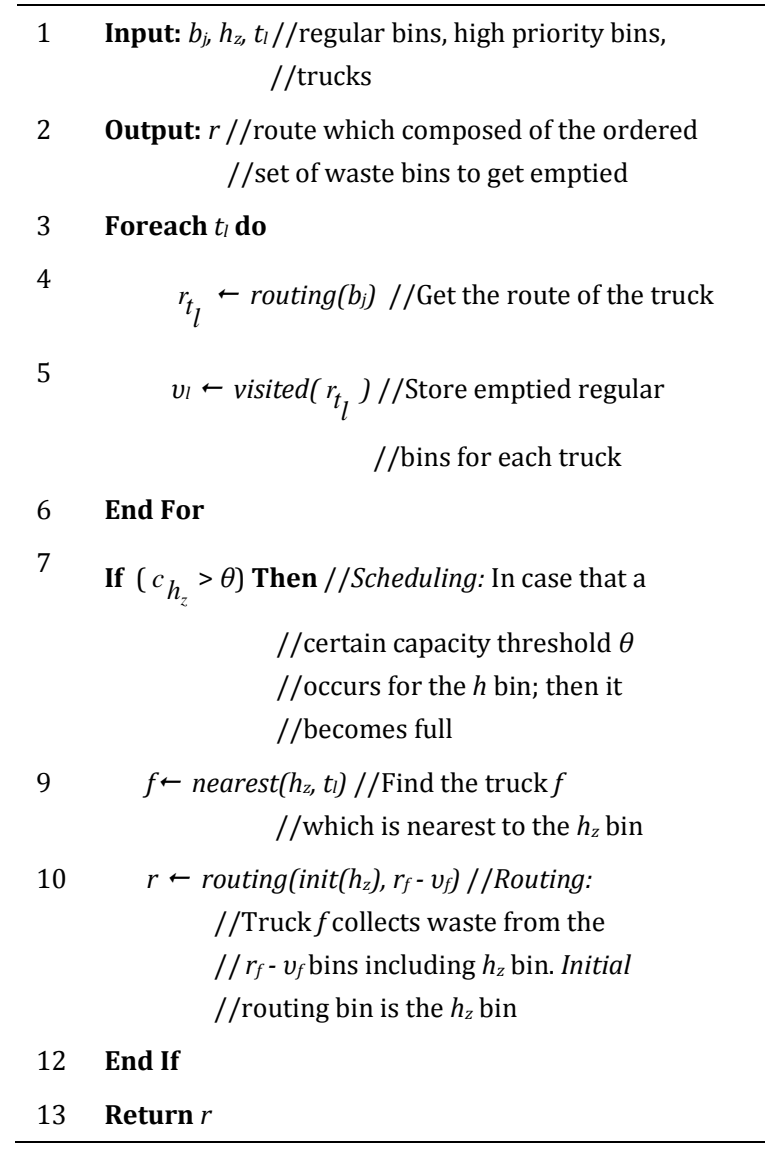

Fig. 5.The MDM algorithm.
The time complexity of the MDM is the complexity of the routing 0 function, which is $O\left(b_{j}{ }^{2}\right)$, plus the complexity of the visited $\left(0\right.$ function, which is $O\left(b_{j}\right)$, the complexity of the init() function which is $O\left(h_{z}\right)$, and the complexity of the nearest $($ function which is $\mathrm{O}\left(h_{z}\right)$. Hence, the overall complexity is $\mathrm{O}\left(b_{j}^{2}\right)$ since $b_{j}$ $>h_{z}$.

\section{The Reassignment Model (RM)}

The last model, the RM, tries to cover the disadvantages of the remaining models and is related to the re-allocation of the sub-areas when an alert arrives to the system. The system takes into consideration the current location of trucks and pays attention on the high priority bins. Specifically, the RM incorporates a reassignment function which reassigns the bins of sub-areas to the trucks' current locations. Accordingly, a nearest location function denotes that routing will select the truck with the nearest location to the location of the specified high priority bin. An initialization function is also incorporated in the routing function of the RM. With this model, we aim to have an up-to-date allocation of the entire area in order to maximize the performance. It should be noted that the re-allocation could result totally different sub-areas compared to the initial produced; however, these results will be fully aligned with the current needs (i.e., alerts defined by high priority bins) and the current location of the trucks. In the re-allocation process, high priority bins are considered in a first served method. Hence, the new routes start with the high priority bins and the rest of the bins follow.

In Fig. 6, we present the proposed RM algorithm. The inputs of the algorithm are the sets of regular, high priority bins and the available trucks. The output is the route for each truck. Initially, the algorithm generates the route of each truck based on the function routing 0 and defines the visited bins with the help of the function visited 0 (set $v_{l}$ ). When a high priority bin becomes full (its capacity $c_{h_{z}}$ is over a threshold $\theta$ ), during the collection process, the algorithm, through the adoption of the reassignment() function creates the new sets of bins (i.e., $\left.\left\{\alpha_{l}\right\}\right)$ devoted to each truck. In these sets, the already visited bins are excluded (i.e., the set $r_{l}-v_{l}$ ). Accordingly, the algorithm finds the nearest truck to the high priority bin produced an alert, through the adoption of the nearest $($ function and the nearest truck $f$ starts its route from the bin $h_{z}$ (i.e., the high priority bin produced an alert). In the re-routing 
process for truck $f$, the algorithm excludes the already visited bins (let $r_{f}-v_{f}$ be the set of the unvisited bins).

In the RM model, the algorithm which performs dynamic re-routing is based on the reassignment algorithm described in (Lim et al., 2005) and the dynamic routing discussed in (Anagnostopoulos et al., 2015a). The algorithm presented in (Lim et al., 2005) is an efficient graph partitioning algorithm based on an implementation of $k$-Means clustering. Specifically, the algorithm is fed up with the set of the remaining bins to be emptied and the available trucks. The locations of the remaining bins are considered to be the clustering data while the trucks' locations are the number of cluster centers. The output of the algorithm is the reassignment of the cluster data (i.e., bins) to certain cluster centers (i.e., trucks). Both, cluster data and cluster centers form certain clusters which are used by the routing0 function.

1 Input: $b_{j}, h_{z}, t_{l} / /$ regular bins, high priority bins,

$$
/ / \text { trucks }
$$

2

Output: $r$ //route which composed of the ordered //set of waste bins to get emptied

3

Foreach $t_{l}$ do

4

$$
r_{t_{l}} \leftarrow \operatorname{routing}\left(b_{j}\right) / / \text { Get the route of the truck }
$$

5

$$
v_{l} \leftarrow \operatorname{visited}\left(r_{t_{l}}\right) / / \text { Store emptied regular }
$$

//bins for each truck

If $\left(c_{h_{z}}>\theta\right)$ Then //Scheduling: In case that a

//certain capacity threshold $\theta$ occurs

$/ /$ for the $h_{z}$ bin; then it becomes full

$\left\{\alpha_{l}\right\} \leftarrow\left\{\right.$ reassignment $\left.\left(t_{l}, r_{l}-v_{l}\right)\right\}$

//Reassigns all the remaining bins to

//the trucks; according to the trucks'

$/ /$ relative positions as in (Lim et al., 2005).

$/ / \alpha_{l}$ is the set of the reassigned

$/ /$ bins to the truck $t_{l}$

9

$f \leftarrow \operatorname{nearest}\left(h_{z}, t_{l}\right) / /$ Find the truck $f$

//which is nearest to the $h_{z}$ bin

10

$r \leftarrow \operatorname{routing}\left(\operatorname{init}\left(h_{z}\right), \alpha_{f}\right) / /$ Routing: Truck $f$

$/ /$ collects waste from the $\alpha_{f}$ bins

//including the $h_{z}$ bin. Initial routing

$/ /$ bin is the $h_{z}$ bin

11 End If

12

Return $r$

Fig. 6. The RM algorithm.
The time complexity of the RM is the complexity of the routing 0 function which is $O\left(b_{j}^{2}\right)$ plus the complexity of the visited $\left(0\right.$ function which is $O\left(b_{j}\right)$, the complexity of the init $\left(0\right.$ function which is $O\left(h_{z}\right)$, the complexity of the nearest $\left(0\right.$ function which is $O\left(h_{z}\right)$ and that of the reassignment $(0$ function which is $\mathrm{O}\left(b_{j}^{2}\right)$. Hence, the overall complexity is $\mathrm{O}\left(b_{j}^{2}\right)$ since $b_{j}$ $>h_{z}$

\section{Application Perspectives}

A WMS typically refers to a specific technique, strategy, or software adopted to manage waste materials. This may include the design of the collection, transportation, recycling, disposal, processing of waste as well as the implementation of such activities. An important part of a WMS is the DSS that mainly concerns scheduling activities. The DSS is responsible to provide schedules for the collection of waste based on a number of criteria. It provides an interface for fleet management and it takes into consideration spatio-temporal characteristics as well as the contextual information of the area under consideration. The DSS realizes the strategy of the local authorities and relevant stakeholders.

The proposed models could be part of a DSS in the following aspects:

- A DSS could be based on a set (pool) of algorithms that perform dynamic adaptation over specific criteria. In the case of high priority areas, the local authorities could define the criteria e.g., cost, distance, response time and the DSS could automatically adopt one of the available models. Our models could be part of the discussed pool. Hence, an intelligent mechanism for selecting the appropriate model for waste collection could be built on top of the discussed algorithms.

- According to the adopted strategy, local authorities could characterize specific areas as high priority and 'force' the system to serve them immediately. This could be done no matter the type of waste (e.g., dangerous materials). For instance, local authorities could select a specific type of waste to be immediately collected to maximize the revenue from recycling. The areas where the specific waste type is mainly disposed (this could be derived by relevant studies) could be defined as high priority areas.

- $\quad$ The proposed models could be adopted by a DSS to manage the trade-off between the immediate collection of high priority bins and the collection costs which are the time spent, the fuel 
consumed and the distance covered by trucks. The DSS could automatically select the model that perfectly matches to pre-defined criteria that are subject to frequent changes. Hence, the WMS could be fully aligned to spatio-temporal criteria. Imagine touristic areas where a large amount of waste is observed only for specific periods (seasonality aspect). Such areas could be characterized as high priority only for the periods where they are crowded.

- $\quad$ The proposed models could be combined with a classification module that processes the available information and will derive classifications for multiple waste types. Hence, the local authorities will be capable of characterizing multiple high priority areas according to the type of waste. The DSS could apply different algorithms for different waste type and, thus, to be fully aligned with the underlying 'waste dynamics' of the SC.

\section{Experimental Evaluation}

We elaborate on the performance of the proposed models i.e., DTM, DM, MDM, RM. Through a set of simulations, we evaluate the performance of each model concerning important metrics that affect the performance of WMSs. We evaluate the proposed models for metrics not only related to the required computational time but also for metrics related to the economic viability of a WMS. The economic viability of a WMS depends on issues like the distance covered by the trucks as well as the required fuels and the time spent in travelling. A number of experimental scenarios are adopted to reveal the advantages and disadvantages of each model. Our simulations are performed for a dataset retrieved for the city of Saint Petersburg, Russia.

The proposed system could be the basis for maximizing the Return of Investment (RoI) from parties involved in the WM chain. There are specific axes through which the RoI could be maximized when we apply the proposed system in a SC. The first is the cost reduction in the waste collection scheme. The proposed models aim to the appropriate management of resources required to the WM and, thus, local authorities taste fuel cost reduction through the optimization of routes (unnecessary transports are minimized). In addition, the efficient management of the collected waste is capable of increasing the material recovery and recycling. When combined with recycling systems will increase the amount of recycled waste with obvious positive impacts in the economic growth of the SC. The percentage of the recycled waste will be maximized accompanied by the economic prosperity of the local societies and the corresponding companies. The above discussed issues are some of the positive impacts that our models have. However, a detailed analysis on the RoI of a waste management system is beyond the scope of this paper.

\section{A. Performance Metrics and Simulation Setup}

We adopt a set of metrics capable of revealing the performance of each model by evaluating quantitative and qualitative characteristics. Quantitative characteristics are related to the quantity of the collected waste, the distance traveled from bins to dumps and the required fuel for delivering the collected waste to dumps. Qualitative characteristics are related to the CPU time required to derive the final routes and the routing time. The list of the adopted metrics is as follows:

- CPU Elapsed Time (CET). The CET metric represents the required time (in seconds) for deriving the final collection result for each model. CET exhibits the time devoted to the definition of the final routes for each truck before the proposed framework's result is applied into the waste collection. The lower the CET is, the better the performance becomes. The reason is that when CET is low, the proposed system does not devote much time to result the final collection route.

- Collected Load (CL). The CL metric depicts the collected volume of waste (in kilograms) that is transported to dumps. The aim is to have high values for the $C L$ metric in order to exploit the entire volume that trucks can transport. In our results, we consider the average $C L$ per truck.

- Distance (D). The $D$ metric measures the trajectory covered (in kilometers) by trucks for delivering waste into the dumps. The aim is to minimize the $D$ metric in order to have a system that results routes involving the shortest paths. In our results, we consider the average $D$ per truck.

- Routing Time (RT). The RT metric represents the time required (in minutes) that trucks need to visit waste bins, perform the collection plan and deliver waste in to the dumps. The $R T$ is not only affected by the $D$ metric but also by qualitative characteristics such as the time required for the actual collection in each bin and the traffic on road network in rush hours.

- Response Time (R). The $R$ metric represents the average time that a truck needs to serve high 
priority bins. The $R$ metric should be low because we aim at delivering a system that immediately serves high priority bins in order to minimize waste consequences in human lives.

- Fuel Quantity (FQ). The $F Q$ metric measures the quantity of fuel (in litters) consumed during the specified routing trips. The $F Q$ depicts the actual economic consequences of each model that finally affects the viability of the proposed waste management system. The aim is to have low values for $F Q$ since this implies economic scaling.

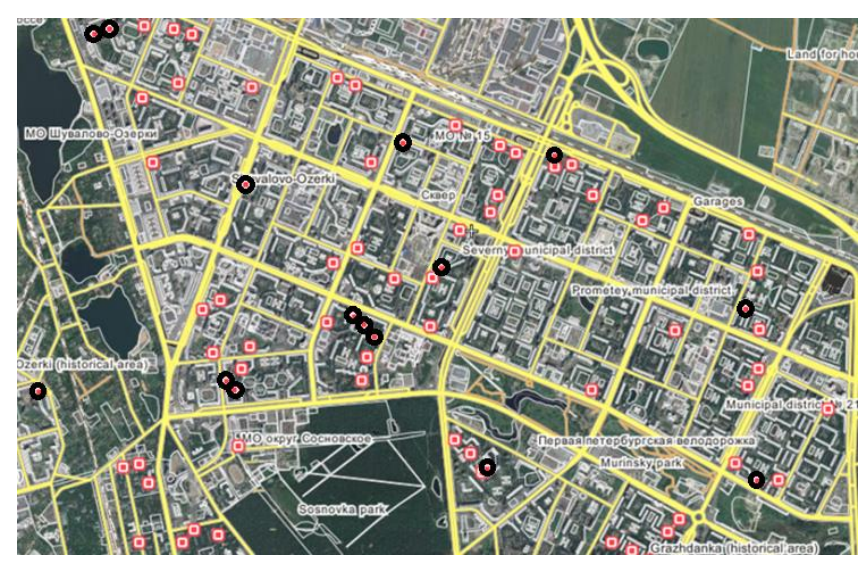

Fig. 7. Distribution of bins (high priority bins are with black) within the municipality of Saint Petersburg, Russia.

Table 1. Simulation parameters and their values.

\begin{tabular}{clc}
\hline Parameter & Description & Value \\
$\boldsymbol{n}$ & The number of sectors & 10 \\
$\boldsymbol{m}$ & The number of regular bins & 250 \\
$\boldsymbol{p}$ & The number of high priority bins & 50 \\
$\boldsymbol{q}$ & The number of trucks in each sector & 6 \\
$\boldsymbol{c}$ & The volume capacity of each bin & $100 \mathrm{Kg}$ \\
$\boldsymbol{C}$ & The volume capacity of each truck & $4000 \mathrm{Kg}$ \\
\hline
\end{tabular}

The proposed models were evaluated with real and synthetic data retrieved by the municipality of Saint Petersburg, Russia ${ }^{3}$. In Fig. 7, we present a real allocation distribution of bins within the municipality. More specifically, the municipality is divided into certain sectors. In Saint Petersburg, for each sector a certain number of regular and high priority bins are assigned as well as a certain number of trucks. This separation is a decision of the Saint Petersburg local authorities for better handling the available waste bins and the fleet of trucks. It should be noted that multiple collection trucks could be devoted to each sector. Each bin as well as each truck has a specific fixed capacity. Table 1 gives more

\footnotetext{
${ }^{3}$ Real allocation distribution of bins within the city of Saint Petersburg. http://wikimapia.org/, [Accessed on: March 9, 2015].
}

details on the adopted setting for the performance evaluation of the proposed models. In our experiments, we also consider variable $\mathrm{n}$ in order to show the performance of our models when sectors number increase. It should be noted that the number of trucks, in total, remains the same which means that for high $\mathrm{n}$ leads to low number of trucks per sector.

We evaluate the proposed models for a period of 30 days (simulation time). Dynamic scheduling is based on the (Anagnostopoulos et al., 2015a) while dynamic routing is achieved with DTM, DM, MDM, and RM models. Specifically, the models are running continuously within that period. A dynamic routing is initialized when a certain threshold of bins is reached based on the dynamic scheduling. The models served, on average, a range of $1-7$ alerts per sector for dynamic routing and per 24 hours. This is natural since some areas are of high priority, thus, require immediate waste collection than the regular areas.

\section{B. Performance Assessment}

Our results for the CET metric are presented in Fig. 8. We observe that the DTM achieves high performance while the RM is the less effective. This is explained since the complexity of the DTM is less than the complexity of the RM. The DTM in contrast to the RM does not contain: (i) a reassignment function, and (ii) a nearest function. The DTM exhibits higher performance than the MDM, as it does not involve a nearest function. Finally, the DTM is more efficient compared to the DM, because in contrast to the DM the routing function does not contain an initialization stage.

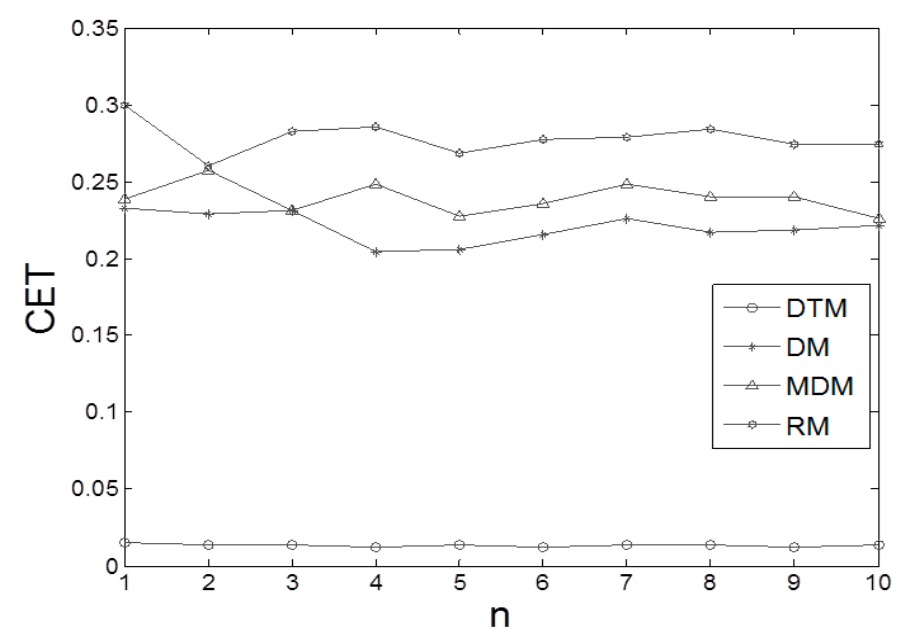

Fig. 8. Results for the CET metric (seconds). 
The $C L$ results for the proposed models are presented in Fig. 9. We can observe that the DTM is less efficient than the remaining models. This is natural since the DTM allocates certain trucks for collecting waste only from the high priority bins. However, the DM, the MDM and The RM allocate the available trucks to collect waste from both regular and high priority bins, thus, exploiting the entire volume capacity of trucks.

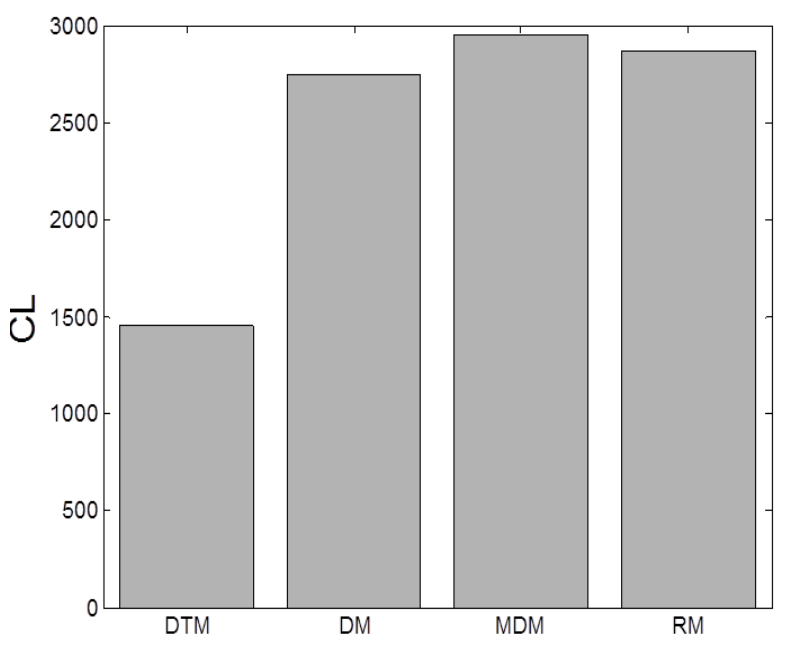

Fig. 9. Results for the $C L$ metric (kilograms).

Our results for the $D$ metric are presented in Table 2. We can observe that the RM is the most efficient model compared to the remaining models. Since the RM incorporates the reassignment function which reallocates routing paths to trucks, the model assigns the unserved bins to the nearest trucks. In the $\mathrm{RM}$, the trajectory covered by trucks for delivering waste into the dumps, after the reassignment function, is minimal, compared to the remaining models. The DM is less efficient since it performs a detour process which leads to maximum trajectories. The MDM exhibits lower performance compared to the rest models and especially to the RM. The MDM calculates the minimum distance between trucks and high priority bins, however, it does not perform any reassignment. Recall that in the MDM, each high priority bin is assigned to the closest collection truck. The DTM performs worse than the MDM, since no calculation of the minimum distance is performed between trucks and high priority bins. It should be noted that our results for the $D$ metric concern the average distance covered by trucks. The $\mathrm{D}$ results cannot be judged as low as we consider that each sector covers an area of 25 square kilometers. Specifically each of the trucks travels $15.8 \mathrm{~km}$ on average which is a sufficient distance for the specified sectors. The bins collected per truck route are in the range of 1 to 50 regular bins and 1 to 10 high priority bins. The range of dynamic routes per day is 1 to 7 adopting the dynamic scheduling.

Table 2. Results for the $D$ metric (kilometers).

\begin{tabular}{ccccc}
\hline Sectors & DTM & DM & MDM & RM \\
$\mathbf{1}$ & 18.7601 & 20.4335 & 18.1074 & 15.8042 \\
$\mathbf{2}$ & 18.4904 & 20.2502 & 17.5486 & 15.9508 \\
$\mathbf{3}$ & 18.0401 & 19.1575 & 18.0812 & 16.5253 \\
$\mathbf{4}$ & 17.8976 & 19.4881 & 17.6272 & 16.6491 \\
$\mathbf{5}$ & 18.2744 & 19.8057 & 17.3739 & 16.1378 \\
$\mathbf{6}$ & 18.5684 & 19.6440 & 17.6719 & 15.2865 \\
$\mathbf{7}$ & 18.5825 & 20.7696 & 18.3960 & 16.3541 \\
$\mathbf{8}$ & 17.7156 & 20.2548 & 17.5711 & 15.7203 \\
$\mathbf{9}$ & 17.9232 & 20.8422 & 18.0638 & 16.1230 \\
$\mathbf{1 0}$ & 18.3599 & 20.0643 & 18.1279 & 17.0431 \\
\hline
\end{tabular}

Our models are also evaluated concerning the RT metric. The RT results are depicted in Fig. 10. The RM performs better than the remaining models. The reason is that the RM derives the lowest $D$ value. In constrast, low $D$ bounds the RT to low values. The DM also exhibits low performance since it has the highest $D$ results. It should be noted that high $D$ leads the $R T$ to high values as well. The DTM and the MDM have similar performance concerning the RT metric, although, they exhibit slightly different $D$ values. The $R T$ metric is not only affected by $D$ but also by qualitative characteristics which have high impact in the $R T$ results. Such qualitative characteristics could be the time required for the actual waste collection and the traffic on road network in rush hours.

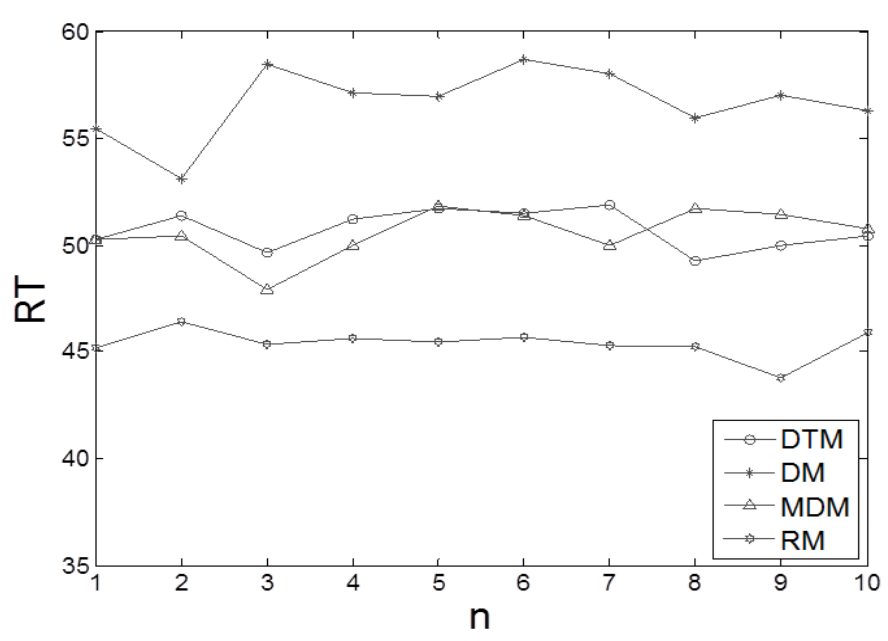

Fig. 10. Results for the $R T$ metric (minutes).

Our results concerning the $F Q$ metric are presented in Fig. 11. The RM achieves high perfrormance since the $F Q$ is highly correlated with $D$ and $R T$ metrics. In the RM results, we observe low 
values for $D$ and $R T$ which, in turn, implies low $F Q$. The DM has the worst performance compared to the remaining models. The reason is that the DM results high $D$ and $R T$ results and, thus, the trucks travel in long distances before they are capable of delivering the collected waste into the dumps. The DTM and the MDM exhibit similar $F Q$ results. However, the MDM is slightly better than the DTM due to the low $D$ results.

We report on the performance of the proposed models for various scenarios concerning the number of high priority bins. Let us denote with $P$ the percentage of high priority bins over the entire set of the available bins. Recall that the number of the available bins in the city of Saint Petersburg is equal to 3000 . Hence, when $p=50 \%$ means that half of the available bins are considered as high priority. With this simulation setup, we try to reveal the performance of the models when the system faces variable high priority bins number. Additionally, we experiment with $\theta=0.8$. Recall that $\theta$ represents the threshold over which a bin is considered as full. In Fig. 12, we present our results concerning the $D$ metric and $p=25 \%$. As $n$ increases, the distance covered by the trucks decreases. This stands for the entire set of the proposed models. The reason is that as $\mathrm{n}$ increases, the area that each sector covers decreases and, thus, a low distance is required for the collection trucks. The RM exhibits the best performance amongst the proposed models followed by the MDM and the DTM. These results are related to the scenario where $\theta=0.8$. In Fig. 13 , we see our results for the $R T$ metric $(p=25 \%)$. In this experimental setting, the RM exhibits the best performance. The increased $\mathrm{n}$ leads to a decreased $R T$ as collection trucks have to cover lower distance compared to scenarios involving low n. In Fig. 14, we present our results for the R metric and for the same experimental scenario $(p=25 \%)$. In this case, the DM has slightly better performance than the RM. The DM leads to $4 \%$ (approximately) less time than the RM in order to serve high priority bins. The worst performance is observed by the MDM.

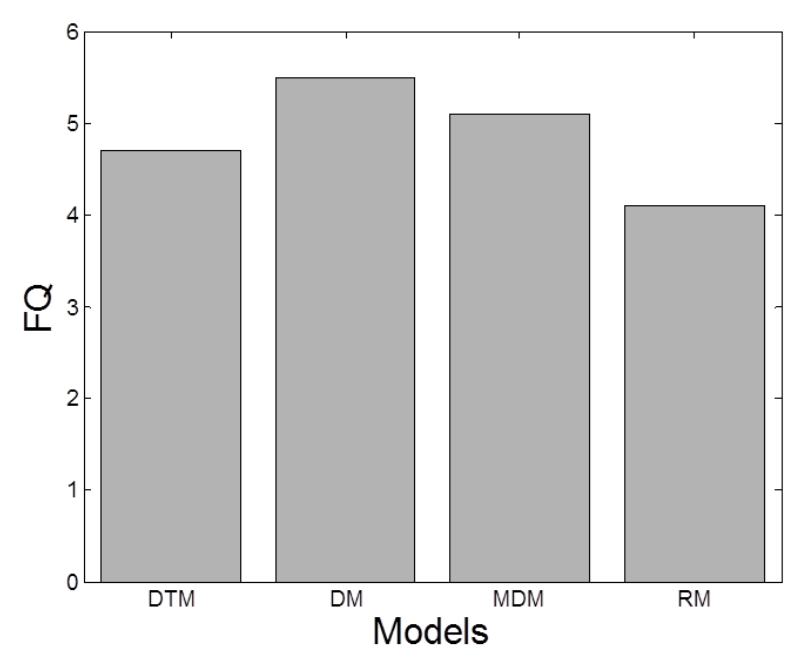

Fig. 11. Results for the $F Q$ metric (litters).

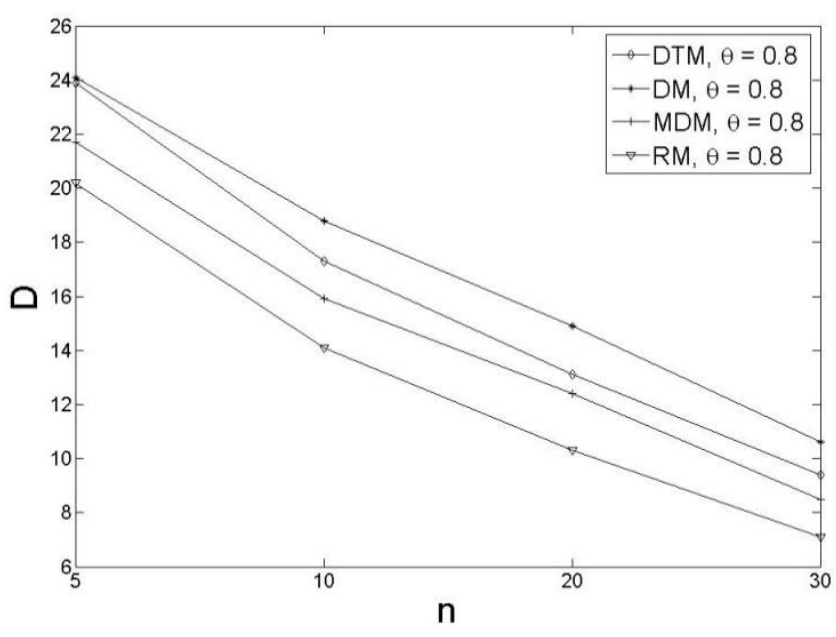

Fig. 12. Performance of the proposed models for $P=25 \%$ ( $D$ metric).

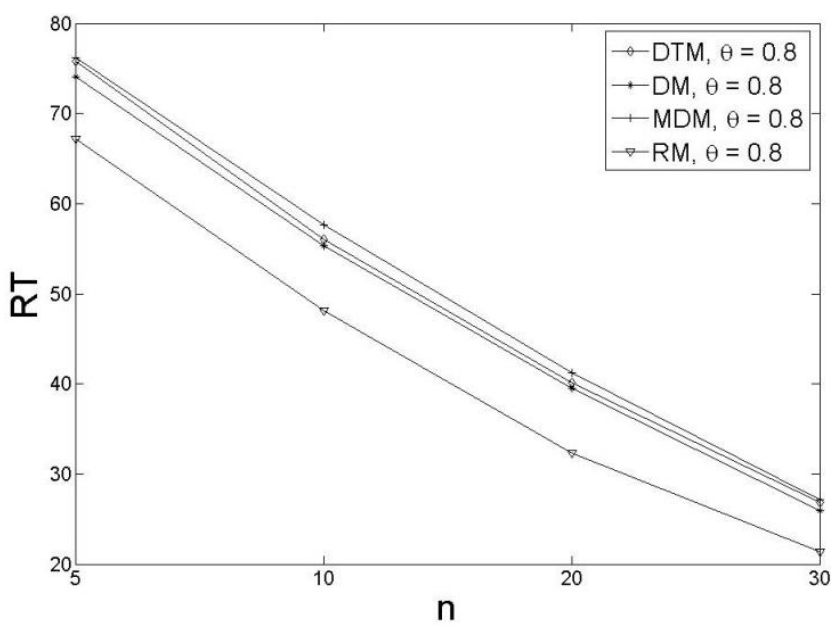

Fig. 13. Performance of the proposed models for $P=25 \%$ (RT metric). 


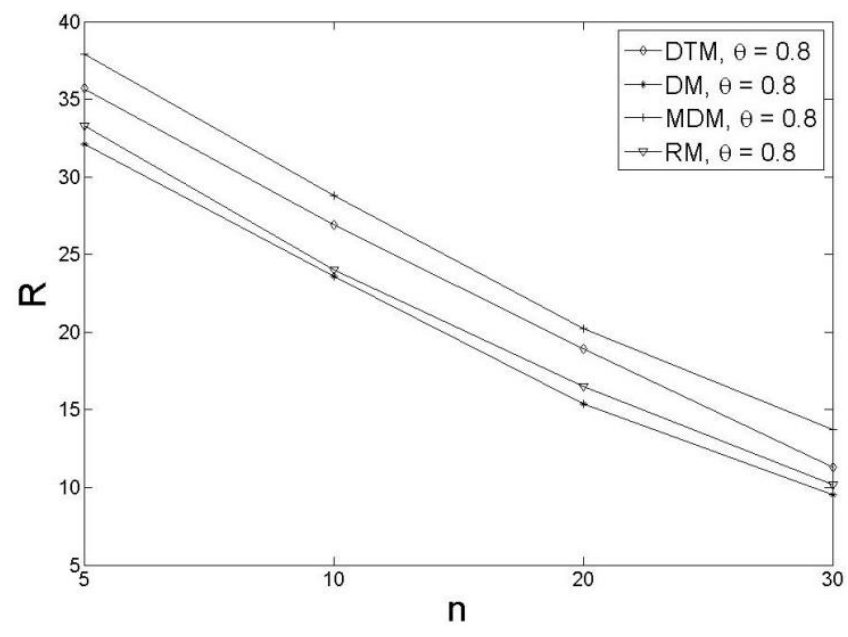

Fig. 14. Performance of the proposed models for $p=25 \%$ ( $R$ metric).

We also perform a set of experiments for $p=50 \%$. In this experimental scenario, we assume that half of the available bins are considered as high priority. In Fig. 15, we see that the lowest distance is covered by the RM and the DTM. When $\mathrm{n} \rightarrow 30$, the DTM exhibits better performance than the remaining models. The DTM seems to be the appropriate model when the area under consideration includes a high percentage of high priority bins. In such cases, it is the best for local authorities to devote specific collection trucks for covering high priority bins. The worst performance is exhibited by the MDM as the model results an increased number of changes in the route of each collection truck. In Fig. 16, we confirm that the $\mathrm{RM}$ requires less routing time compared to the remaining models while the MDM exhibits the worst performance. As far as the $\mathrm{R}$ metric concerns, our results depicted in Fig. 17 show that the DTM is the best model if local authorities want to achieve limited time for serving high priority bins. Recall that we assume an area with a high number of high priority bins. In such cases, the RM results the lowest routing time, however, the model is not appropriate to immediately serve high priority bins. Finally, the $R$ decreases as $n$ increases. The reason is that a high number of sectors lead to small areas that should be served by the collection trucks. However, in such cases, the trucks should cover a low number of bins.

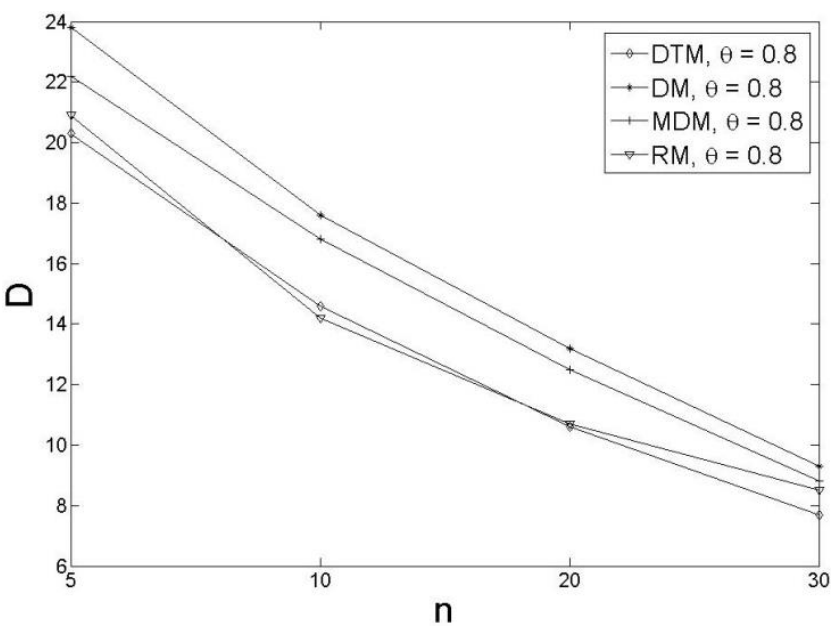

Fig. 15. Performance of the proposed models for $p=50 \%$ ( $D$ metric).

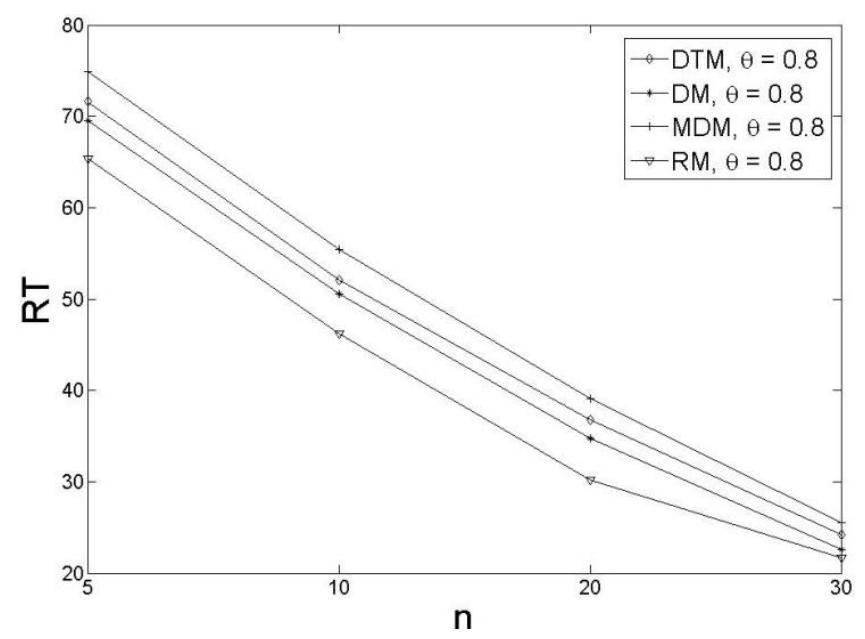

Fig. 16. Performance of the proposed models for $p=50 \%$ (RT metric).

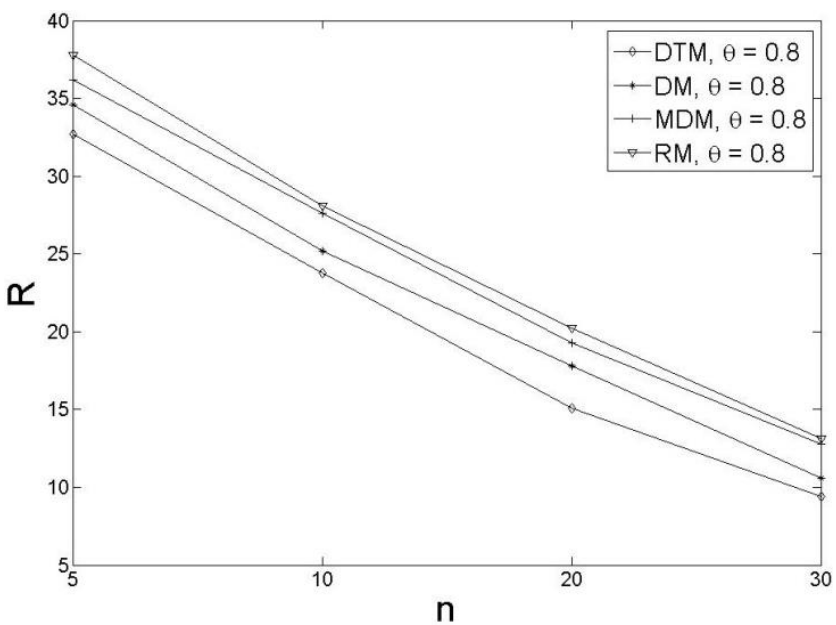

Fig. 17. Performance of the proposed models for $p=50 \%$ ( $R$ metric).

We perform additional experiments for different values of $q$ and $C$. The aim is to reveal the performance of the proposed models when the 
number of trucks in each sector and their capacity change. In experiments, we get $q \in\{4,6,10,20\}, C \in$ $\{2000,3000,4000,5000\}(\mathrm{Kg})$ and $p \in\{5 \%, 50 \%\}$ while keeping $n=10$. We report on the $D, R T$ and $R$ results.

In Figs 18, 19 and 20, we see our results for $p=$ $5 \%$ and different $q$. In general, the proposed models result similar $D$ values (Fig. 18). As the number of trucks increases, the distance that they cover, naturally, decreases. We observe similar decrease in the routing $(R T)$ and the response time $(R)$ (Figs 19, 20 ). Multiple trucks could easily serve not only high priority bins but also regular bins. In such cases, the trucks devote low time for routing. The RM exhibits the best and the DM exhibits the worst performance for both, the $R T$ and the $R$ metrics.

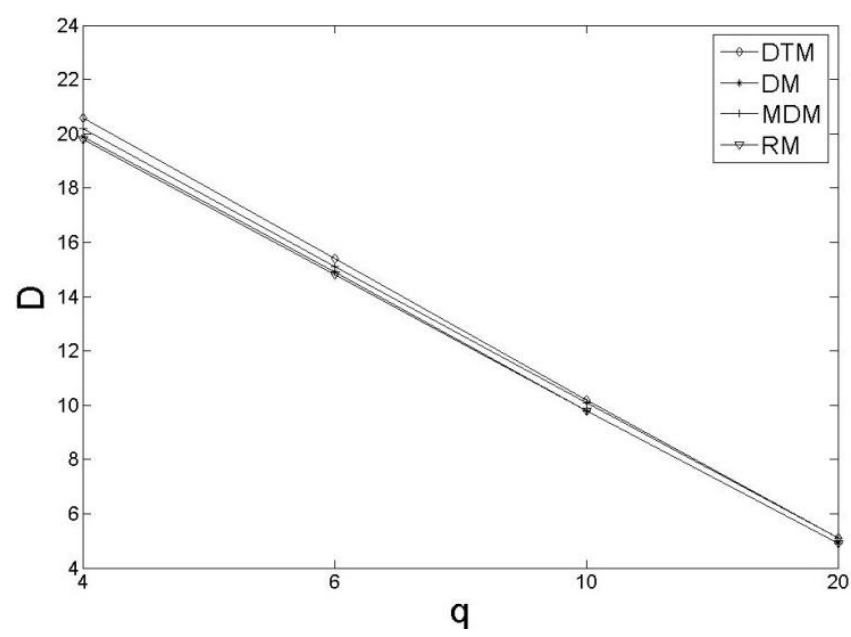

Fig. 18. Performance of the proposed models for $p=5 \%$ and different $q$ ( $D$ metric).

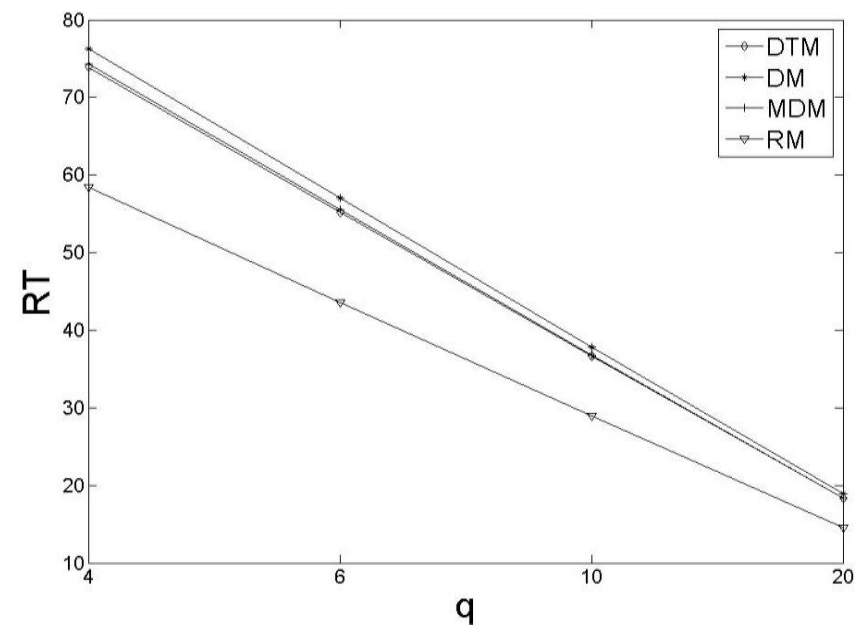

Fig. 19. Performance of the proposed models for $p=5 \%$ and different $q$ (RT metric).

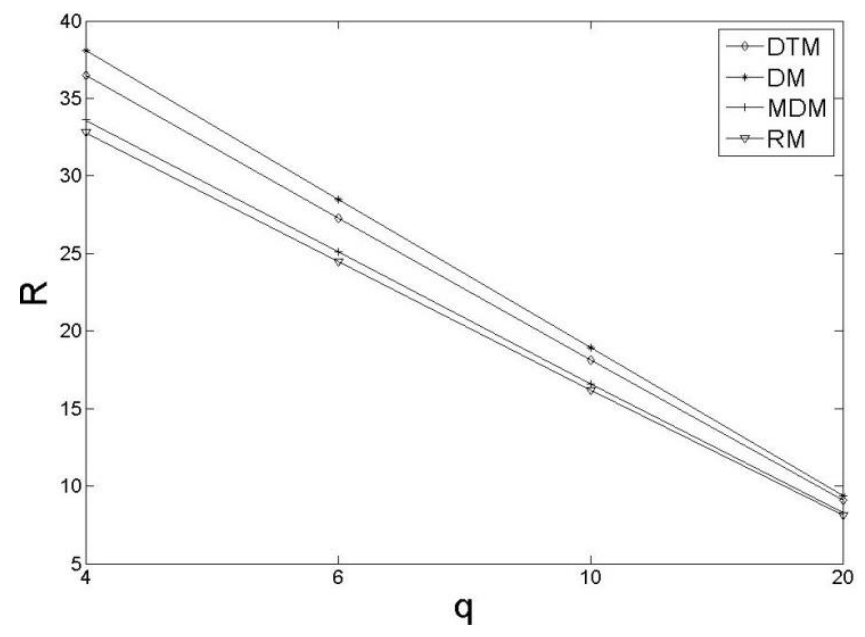

Fig. 20. Performance of the proposed models for $p=5 \%$ and different $q$ ( $R$ metric).

In Figs 21, 22 and 23, we see our results for $p=$ $50 \%$ and different $q$. In this scenario, the proposed models cover high distances (Fig. 21) when $q \rightarrow 4$ compared to the experimental scenario where $p=$ $5 \%$. This is reasonable, as in the scenario where $p=$ $50 \%$, the available trucks are limited and, thus, they should cover high distances to serve the high number of high priority bins. These results mainly stand for the DM and the MDM. Concerning the RT metric (Fig. 22 ), we get similar results as in the scenario where $p$ $=5 \%$. However, now, the worst performance is exhibited by the MDM instead of the DM. The RM remains the model with the best performance. Moreover, we observe additional differences in the performance of the proposed models related to the $\mathrm{R}$ metric with the previously discussed experimental scenario $(p=5 \%)$. When $p=50 \%$, the DTM exhibits the best performance concerning the $R$ metric (Fig. 23) and the RM the worst. Now, we have a high number of high priority bins that should be immediately served. The DTM devoting specific trucks to the high priority bins is the model that should selected in such cases as it is the model exhibiting the lowest response time. 


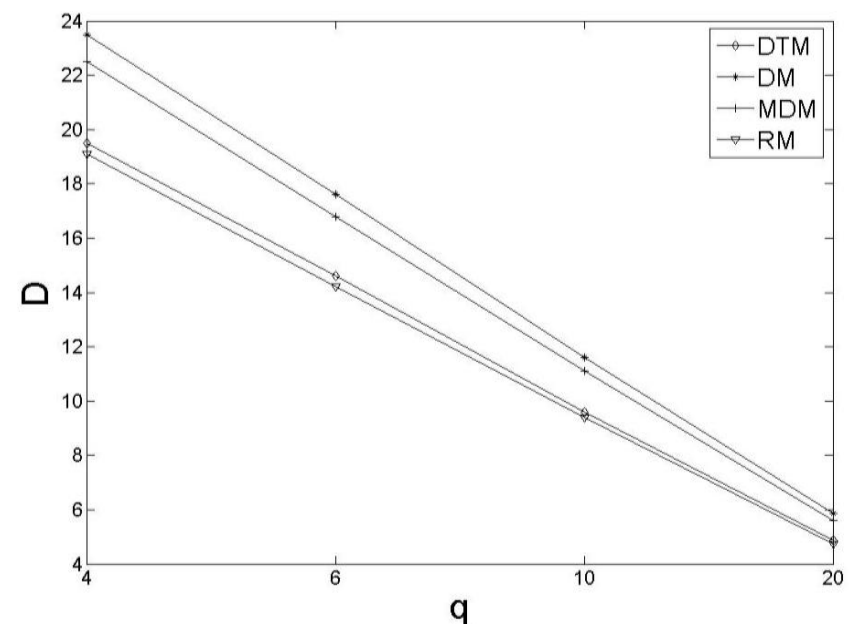

Fig. 21. Performance of the proposed models for $p=50 \%$ and different $q$ ( $D$ metric).

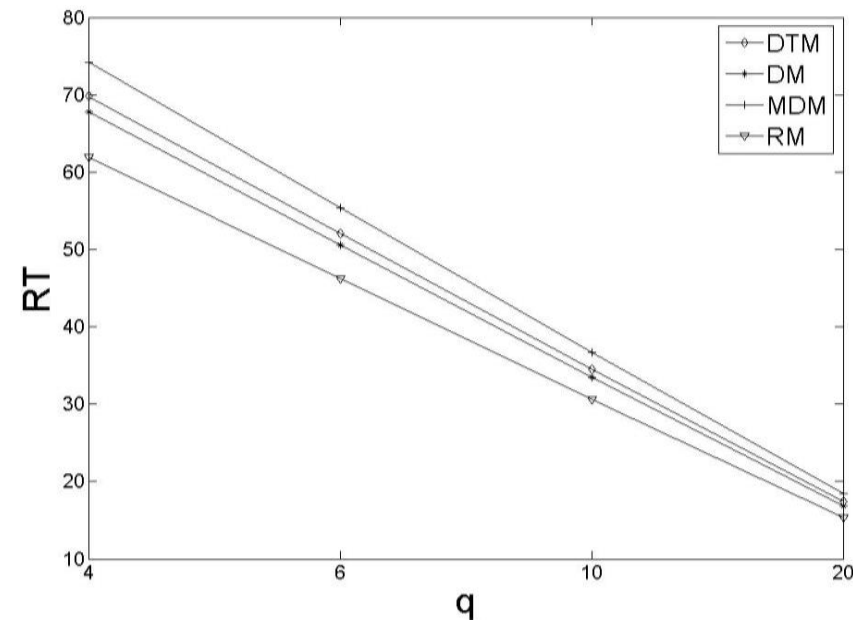

Fig. 22. Performance of the proposed models for $p=50 \%$ and different $q$ ( $R T$ metric).

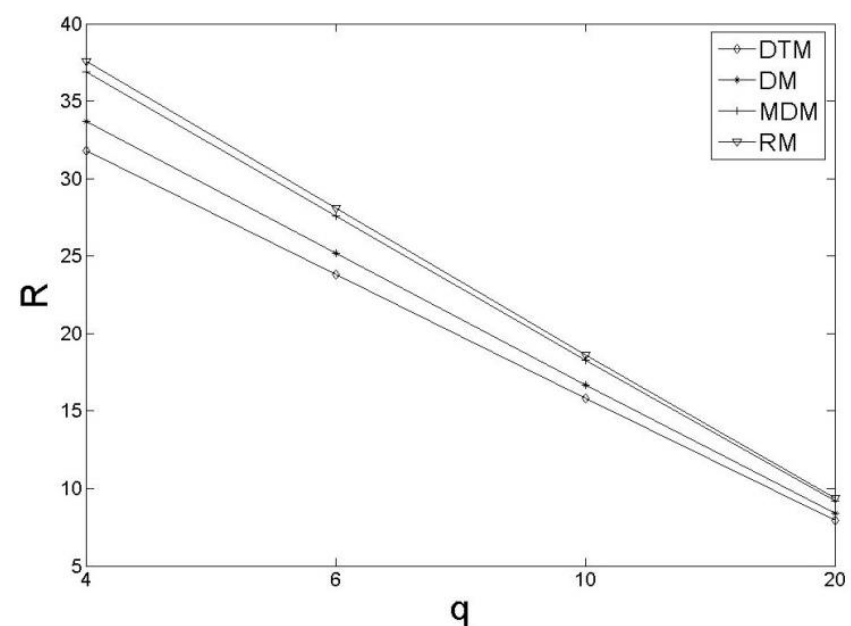

Fig. 23. Performance of the proposed models for $p=50 \%$ and different $q$ ( $R$ metric).

In Figs 24, 25 and 26, we depict our results for different $C(C \in\{2000,3000,4000,5000\})$ and $p=$ $5 \%$. We aim to present the performance of the proposed models when the capacity of the available trucks changes. Concerning the $D$ metric, the proposed models exhibit similar performance, however, the distance increases as the capacity increases. The reason is that each truck when it has an increased capacity can collect more waste before it is ready to be guided in the disposal area. The routing time ( $R T$ metric) also increases as $C \rightarrow 5000$. This is reasonable as the distance increases and each truck spends more time for the collection process. The best performance is observed for the RM while the worst performance is observed for the DM. Similar results can be seen for the $R$ metric.

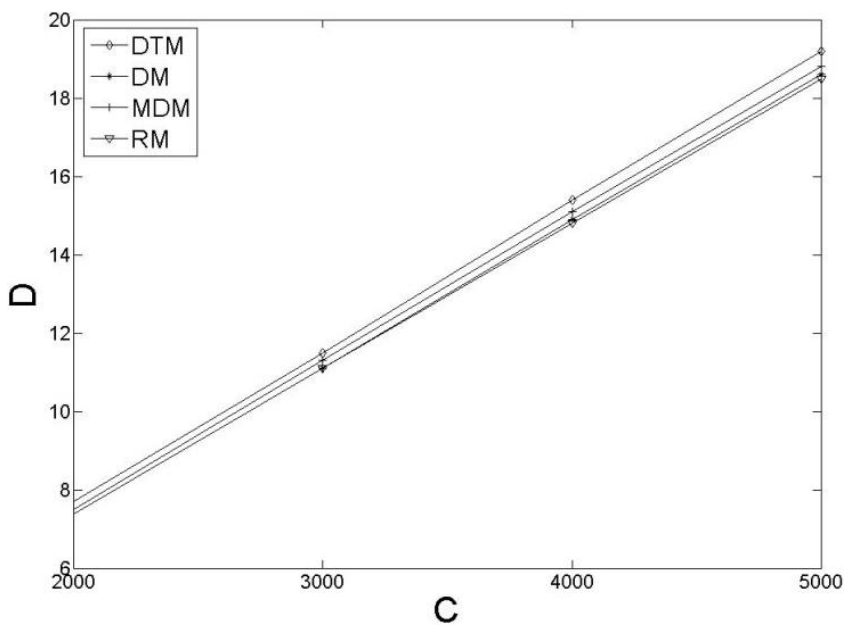

Fig. 24. Performance of the proposed models for $p=5 \%$ and different $C$ ( $D$ metric).

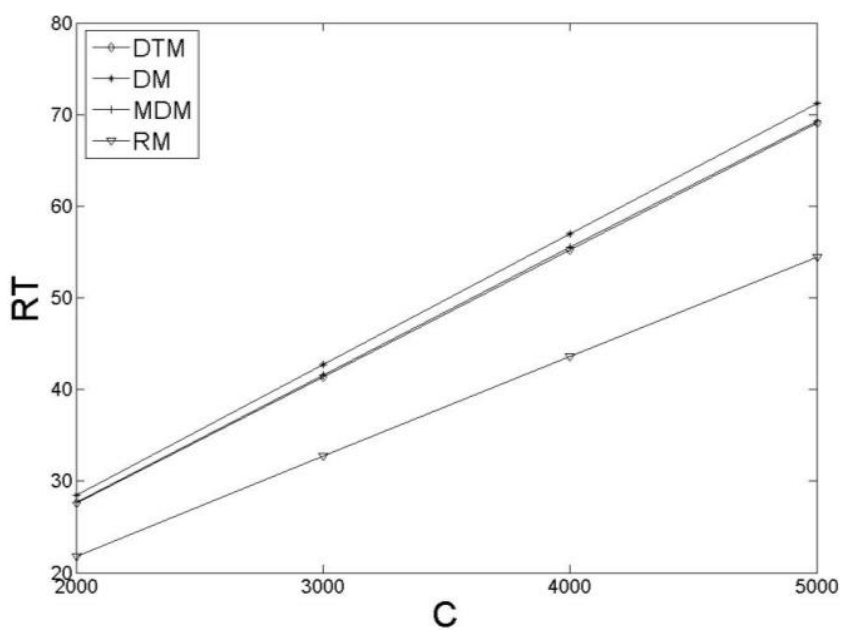

Fig. 25. Performance of the proposed models for $p=5 \%$ and different $C$ (RT metric). 


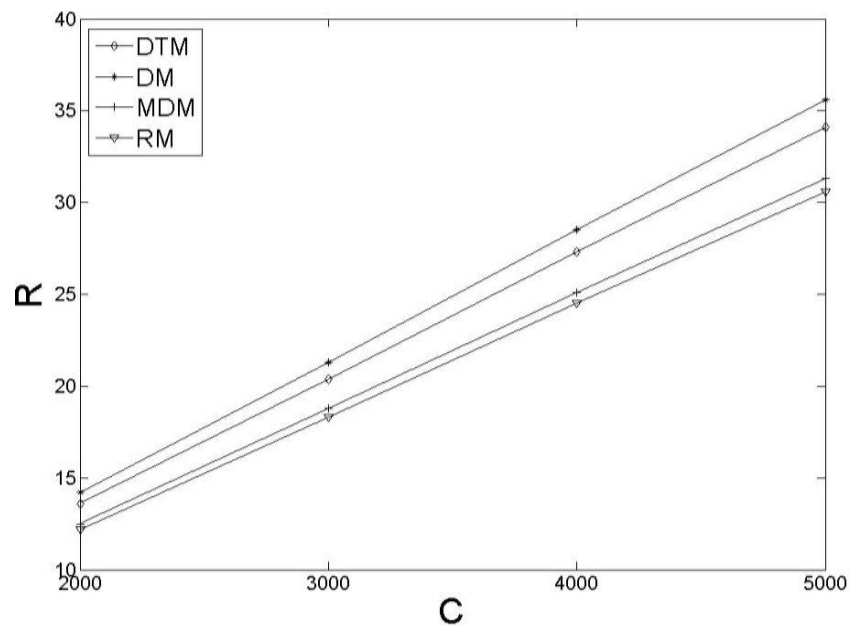

Fig. 26. Performance of the proposed models for $p=5 \%$ and different $C$ ( $R$ metric).

In addition, we get $p=50 \%$ to simulate a high number of high priority bins. In Figs 24, 25 and 26, we depict our results for different $C$. The RM and the DM exhibit the lowest and the highest distance, respectively (Fig. 24). In Fig. 25, we observe that the RM remains the model with the best performance and the MDM is the model with the worst. These results stand for the $R T$ metric. Moreover, concerning the metric $R$, we confirm the results depicted in Fig. 23. As we see in Fig. 26, the DTM exhibits the lowest response time while the RM the highest. These results indicate that when the number of high priority bins is large, the public authorities should devote specific trucks to serve high priority areas / bins.

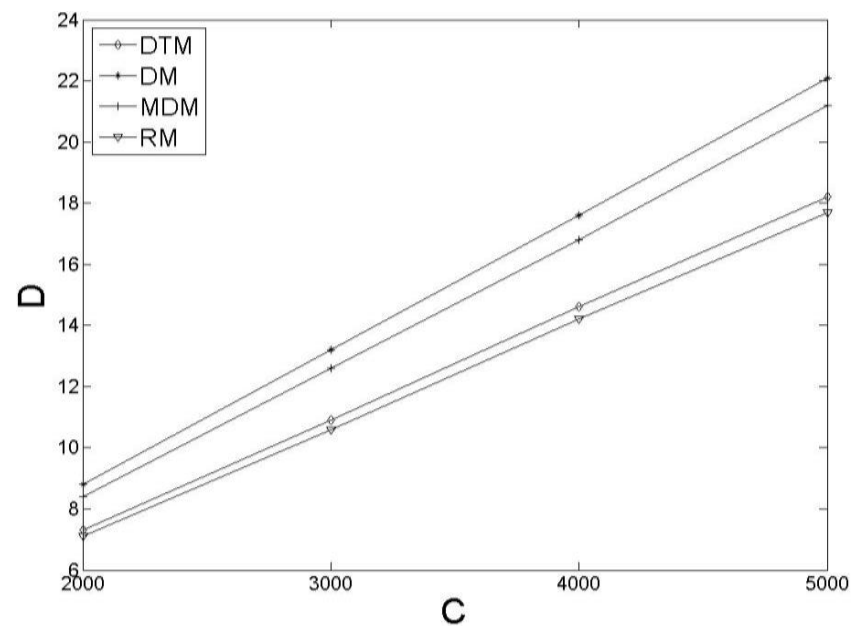

Fig. 27. Performance of the proposed models for $p=50 \%$ and different $C$ ( $D$ metric).

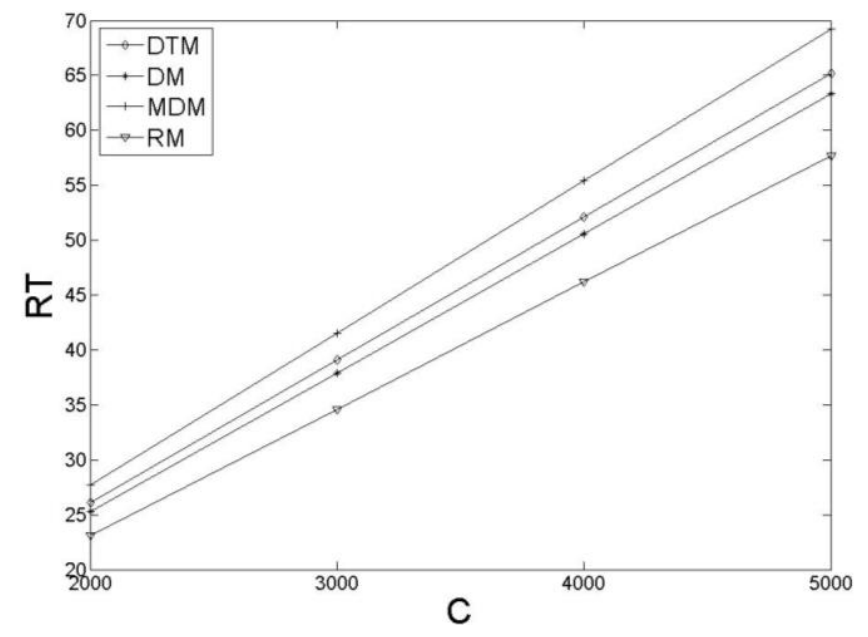

Fig. 28. Performance of the proposed models for $p=50 \%$ and different $C$ (RT metric).

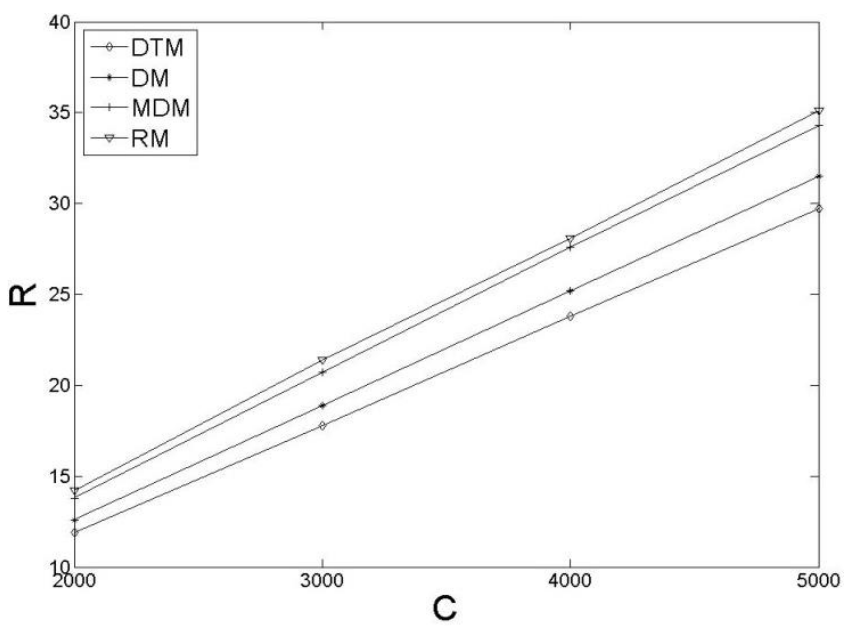

Fig. 29. Performance of the proposed models for $p=50 \%$ and different $C$ ( $R$ metric).

Finally, in Table 3 and Table 4, we summarize the cases where each model performs well. The tables are provided in order to help developers and stakeholders to choose between the proposed models. It should be noted that in Table 3, we get the average case retrieved by the entire set of our experiments and classify the performance in the set \{Low, Medium, High $\}$.

Table 3. Models Performance.

\begin{tabular}{c|cccccc}
\hline & \multicolumn{5}{c}{ Metric } \\
Model & $\boldsymbol{C E} \boldsymbol{T}$ & $\boldsymbol{C L}$ & $\boldsymbol{D}$ & $\boldsymbol{R} \boldsymbol{T}$ & $\boldsymbol{R}$ & $\boldsymbol{F Q}$ \\
\hline DTM & Low & Low & Medium & Medium & Low & Medium \\
DM & High & High & High & Medium & Medium & High \\
MDM & High & High & Medium & Medium & Medium & High \\
RM & High & High & Low & Low & Medium & Low \\
\hline
\end{tabular}

Table 4. Performance Domains. 


\begin{tabular}{cccccc}
\hline Model & $\begin{array}{c}\text { Dedicated } \\
\text { Trucks per } \\
\text { bins Type }\end{array}$ & $\begin{array}{c}\text { Dedicated } \\
\text { trucks per } \\
\text { Sector }\end{array}$ & $\begin{array}{c}\text { Same } \\
\text { Sector } \\
\text { Support }\end{array}$ & $\begin{array}{c}\text { Cross } \\
\text { Sector } \\
\text { Support }\end{array}$ & $\begin{array}{c}\text { Re- } \\
\text { Assignment } \\
\text { Support }\end{array}$ \\
DTM & $\checkmark$ & & $\checkmark$ & $\checkmark$ & \\
DM & & $\checkmark$ & $\checkmark$ & & \\
MDM & & $\checkmark$ & $\checkmark$ & $\checkmark$ & \\
RM & & & $\checkmark$ & $\checkmark$ & $\checkmark$ \\
\hline
\end{tabular}

\section{Conclusions and Future Work}

The immediate collection of waste from high priority bins is a challenging problem in modern societies. The reason is that, nowadays, due to the increased population accompanied by the industrial development, the probability of exposing dangerous waste to citizens is increased as well. Especially, in the cases where waste is dangerous for human lives or for specific parts of the population, the need for the immediate collection is imperative. We propose a set of models for alleviating the discussed problem. The proposed models deal with specific strategies for serving high priority bins. All of them aim to cover specific aspects of the problem. Local authorities or stakeholders could adopt a model to be applied in real scenarios. A high number of simulations reveal the advantages and disadvantages of the proposed models. We report on the performance of each model for a wide set of metrics. These metrics deal with quantitative as well as qualitative characteristics of a waste management system.

In the first places of our future research agenda is the definition of an intelligent mechanism for the management of historical data related to the load of high priority bins. Hence, our system will be capable of providing pro-active responses in the demand for collecting waste from high priority bins. Pro-active responses will increase the efficiency of the system as they will be the basis of building novel routing algorithms that incorporate such knowledge in their results. For instance, spatio-temporal data combined with bins load historical data will give us the opportunity to derive routes that, in specific hours of the day and for specific sectors of the city, will give priority to the discussed bins. Finally, a dynamic reallocation of routes according to the load of each truck will be another extension of our work. Through this approach, borders between sectors will be eliminated and, if necessary, trucks will undertake the responsibility of collecting waste in their 'neighbors'.

\section{Acknowledgements}

The research was carried out with the financial support of the Ministry of Education and Science of the Russian Federation under grant agreement \#14.575.21.0058.

\section{References}

Anagnostopoulos, T., Zaslavsky, A., 2014. Effective Waste Collection with Shortest Path Semi-Static and Dynamic Routing. Internet of Things, Smart Spaces and Next Generation Networks and Systems, Lecture Notes in Computer Science, vol. 8638, pp. 95105.

Anagnostopoulos, T., Zaslavsky, A., Medvedev, A., 2015a. Robust Waste Collection exploiting Cost Efficiency of IoT potentiality in Smart Cities. IEEE $1^{\text {st }}$ International Conference on Recent Advances in Internet of Things (RIoT), Singapore, April, pp. 1-6.

Anagnostopoulos, T., Zaslavsky, A., Medvedev, A., Khoruzhnikov, S., 2015b. Top-k Query based Dynamic Scheduling for IoT-enabled Smart City Waste Collection. In Proc. of the 16th IEEE International Conference on Mobile Data Management (MDM 2015), Pittsburgh, US.

Bakici, T., Almirall, E., Wareham, J., 2013. A Smart City Initiative: The Case of Barcelona. Journal of Knowledge Economy, vol. 4(2), pp. 135-148.

Bing, X., 2014. Vehicle routing for the eco-efficient collection of household plastic waste. Waste Management, vol. 34 (4), pp. 719729.

Buhrkal, K., Larsen, A., Ropke, S., 2012. The Waste Collection Vehicle Routing Problem with Time Windows in a City Logistics Context. Procedia Social and Behavioral Sciences, vol. 39, pp. 241254.

Centre of Regional Science, 2015. Vienna University of Technology. Smart Cities. Ranking of European Medium-Sized Cities. http://www.smart-cities.eu, [Accessed on: February 18, 2015].

Hemmelmayr, V., Doerner, K. F., Hartl, R. F., Rath, S., 2013. A heuristic solution method for node routing based solid waste collection problems. Journal of Heuristics, vol. 19(2), pp. 129-156.

Jin, J., Gubbi, J., Marusic, S., Palaniswami, M., 2014. An Information Framework for Creating a Smart City through Internet of Things. Internet of Things Journal, vol. 1(2), pp. 112-121.

Johansson, O. M., 2006. The Effect of Dynamic Scheduling and Routing in a Solid Waste Management System. Waste Management, vol. 26(8), pp. 875-885.

Li, J. Q., Borenstein, D., Mirchandani, P. B., 2008. Truck Scheduling for Solid Waste Collection in the City of Porto Alegre, Brazil. Omega, vol. 36(6), pp. 1133-1149.

Lim, C., Bohacek, S., Hespanha, J. P., Obraczka, K., 2005. Hierarchical Max-Flow Routing. In Proceedings of the IEEE Global Telecommunications Conference (GLOBECOM), pp. 550-556.

Mes, M., 2012. Using Simulation to Assess the Opportunities of Dynamic Waste Collection. Use Cases of Discrete Event Simulation, pp. 277-307. 
Mes, M., Schutten, M., Rivera, A. P., 2013. Inventory routing for dynamic waste collection. Beta conference, WP No. 431, Eindhoven, Netherlands.

Milić, P., Jovanović, M., 2011. The Advanced System for Dynamic Vehicle Routing in the Process of Waste Collection. FactaUniversitatis, Series: Mechanical Engineering, vol. 9(1), pp. 127-136.

Minghua, Z., Xiumin, F., Rovetta, F., Qichang, H., Vicentini, H., Bingkai, L., Giusti, A., Yi, L., 2009. Municipal solid waste management in Pudong New Area, China. Waste Management, vol. 29(3), pp. 1227-1233.

Minh, T. T., Hoai, T. V., Nguyet, T. T. N., 2013. A Memetic Algorithm for Waste Collection Vehicle Routing Problem with Time Windows and Conflicts. Computational Science and Its Applications, ICCSA, Lecture Notes in Computer Science, vol. 7971, pp. 485-499.

Nadizadeh, A., Nasab, H. H., 2014. Solving the Dynamic Capacitated Location-Routing Problem with Fuzzy Demands by Hybrid Heuristic Algorithm. European Journal of Operational Research, vol. 238(2), pp. 458-470.

Nam, T., Pardo, T. A., 2012. Conceptualizing Smart City with Dimensions of Technology, People and Institutions. In Proceedings of the 12th Annual ACM International Digital Government Research Conference: Digital Government Innovation in Challenging Times, pp. 282-291.

Nuortio, T., Kytojoki, J., Niska H., Braysy, O., 2006. Improved route planning and scheduling of waste collection and transport. Expert Systems with Applications, vol. 30(2), pp. 223-232.

Priano, F. H., Guerra, C. F., 2014. A Framework for Measuring Smart Cities. In Proceedings of the 15th ACM Annual International Conference on Digital Government Research, pp. 44-54.

Ramos, T. R. P., Gomes, M. I., Barbosa-Povoa, A. P., 2014.Assessing and Improving Management Practices when Planning Packaging Waste Collection Systems. Resources Conservation and Recycling, vol. 85, pp. 116-129.

Reed, M., Yiannakou, A., Evering, R., 2014. An ant colony algorithm for the multi-compartment vehicle routing problem. Applied Soft Computing, vol. 15, pp. 169-176.

Stellingwerff, A., 2011. Dynamic Waste Collection: Assessing the Usage of Dynamic Routing Methodologies. Master Thesis, Industrial Engineering \& Management, University of Twente, Twente Milieu.

Vermesan, O., Friess, P., 2013. Internet of Things: Converging Technologies for Smart Environments and Integrated Ecosystems. River Publishers, Aalborg, Denmark.

Von Poser I., Awad, A. R., 2006. Optimal Routing for Solid Waste Collection in Cities by using Real Genetic Algorithm. Information and Communication Technologies, ICTTA, vol. 1, pp. 221-226.

Wy, J., Kim, B.-I., Kim, S., 2013. The rollon-rolloff waste collection vehicle routing problem with time windows. European Journal of Operational Research, vol. 224(3), pp. 466-476.

Zsigraiova, Z., Semiao, V., Beijoco, F., 2013. Operation Costs and Pollutant Emissions Reduction by Definition of new Collection Scheduling and Optimization of MSW Collection Routes using GIS.
The Case Study of Barreiro, Portugal. Waste Management, vol. 33(4), pp. 793-806. 